Revue internationale P.M.E.

Économie et gestion de la petite et moyenne entreprise

Revure

internationale

PME

\title{
La conservation des ressources humaines en PME
}

\section{Denis J. Garand et Bruno Fabi}

Volume 7, numéro 1, 1994

URI : https://id.erudit.org/iderudit/1008371ar

DOI : https://doi.org/10.7202/1008371ar

Aller au sommaire du numéro

\section{Éditeur(s)}

Presses de l’Université du Québec

ISSN

0776-5436 (imprimé)

1918-9699 (numérique)

Découvrir la revue

Citer cet article

Garand, D. J. \& Fabi, B. (1994). La conservation des ressources humaines en PME. Revue internationale P.M.E., 7(1), 85-126.

https://doi.org/10.7202/1008371ar

\section{Résumé de l'article}

Deuxième d'une trilogie consacrée à la gestion des ressources humaines (GRH) dans les PME, cet article résume une démarche visant à décrire et à commenter les principales pratiques de conservation des ressources humaines en contexte de PME. Pour y parvenir, l'expérience internationale a été analysée à travers la documentation empirique rapportant des résultats de terrain dans divers pays d'Europe et d'Amérique du Nord. Une première constatation révèle que, dans l'ensemble, la rémunération et les avantages sociaux représentent les activités de GRH les plus formalisées par des politiques et des procédures puisqu'une proportion variant entre 45 et $80 \%$ de PME formalisent ces pratiques, les pourcentages augmentant avec la taille de l'organisation et le niveau de syndicalisation. Les propriétaires-dirigeants de PME accordent en effet une attention particulière à ces activités de GRH puisqu'ils sont constamment confrontés au problème suivant: le danger de perdre des ressources humaines hautement qualifiées au profit de concurrents de plus grande taille disposés à offrir des conditions financières plus alléchantes. Cette situation de concurrence, avec plusieurs autres facteurs de contingence, explique en partie le recours croissant à des formes plus complexes d'incitation et d'intéressement, bien que ces méthodes demeurent facultatives en France (pour les PE de moins de 100 salariés) et dans la plupart des pays industrialisés. Par ailleurs, il en va tout autrement pour ¡'évaluation du rendement, qui s’avère nettement moins formalisée dans les PME. Avec la planification des ressources humaines et l'analyse des emplois, elle constitue la pratique la moins structurée et la plus galvaudée par les propriétaires-dirigeants qui effectuent bien souvent eux-mêmes une appréciation paternaliste des performances de leurs employés. Comme pour la rémunération, le niveau de formalisation de l'évaluation du rendement est influencé par la taille organisationnelle ainsi que par la syndicalisation. C'est ainsi que les politiques écrites d'évaluation du rendement sont trois fois plus fréquentes dans les organisations de 150 employés, comparativement aux TPE de moins de 25 personnes. Les critères d'évaluation retenus concernent habituellement la personne et ses résultats plutôt que des comportements professionnels, difficiles à définir et à observer de façon continue. Bref, les pratiques de conservation des ressources humaines, bien que généralement peu formalisées, existent à des degrés fort variables, faisant ainsi encore ressortir le caractère fortement hétérogène des PME, limitant dès lors toute généralisation concernant les pratiques de GRH qu'on y retrouve.
Ce document est protégé par la loi sur le droit d'auteur. L'utilisation des services d'Érudit (y compris la reproduction) est assujettie à sa politique d'utilisation que vous pouvez consulter en ligne.

https://apropos.erudit.org/fr/usagers/politique-dutilisation/ 


\title{
La conservation des ressources humaines en PME
}

\author{
Denis J. GARAND* \\ Université du Québec en Abitibi-Témiscamingue \\ Bruno $\left.F A B\right|^{\star *}$ \\ Université du Québec à Trois-Rivières
}

\begin{abstract}
RÉSUMÉ
Deuxième d'une trilogie consacrée à la gestion des ressources humaines (GRH) dans les PME, cet article résume une démarche visant à décrire et à commenter les principales pratiques de conservation des ressources humaines en contexte de PME. Pour y parvenir, l'expérience internationale a été analysée à travers la documentation empirique rapportant des résultats de terrain dans divers pays d'Europe et d'Amérique du Nord. Une première constatation révèle que, dans l'ensemble, la rémunération et les avantages sociaux représentent les activités de GRH les plus formalisées par des politiques et des procédures puisqu'une proportion variant entre 45 et $80 \%$ de PME formalisent ces pratiques, les pourcentages augmentant avec la taille de l'organisation et
\end{abstract}

* Denis J. Garand est chargé d'enseignement en management à l'Université du Québec en Abitibi-Témiscamingue (Rouyn-Noranda - UQAT). Il détient une maîtrise de recherche (M. Sc.) en gestion de PME de l'UQTR et a collaboré à plusieurs projets de recherche appliqués aux petites entreprises. Collaborateur externe du GREPME, ses travaux portent sur les pratiques de GRH des PME et des entreprises œuvrant en gestion de projets, leur degré de formalisation et l'impact de certains facteurs de contingence : taille organisationnelle, propriétaire-dirigeant, vision entrepreneuriale, présence syndicale, orientations stratégiques et niveau de sophistication technologique. Adresse: 371, avenue Bellevue, Laval (Québec) H7C 1T4, tél. et télécopie: (514) 661-9018.

** Bruno Fabi est professeur agrégé à l'UQTR et coordonne les cours de GRH aux premier et deuxième cycles. Il est titulaire d'un doctorat (D. Ps.) en psychologie industrielle et organisationnelle de l'Université de Montréal, et est l'auteur de plusieurs ouvrages, articles académiques et professionnels. Ses intérêts de recherche et d'intervention portent principalement sur la GRH, la gestion participative et le développement organisationnel. Membre associé du GREPME, il agit également à titre d'évaluateur pour divers périodiques et organismes subventionnaires. Adresse : Département d'administration et d'économique, Université du Québec à Trois-Rivières, C.P. 500, Trois-Rivières (Québec) G9A 5H7, tél. : (819) 376-5080; télécopie: (819) 376-5079. 
le niveau de syndicalisation. Les propriétaires-dirigeants de PME accordent en effet une attention particulière à ces activités de GRH puisqu'ils sont constamment confrontés au problème suivant: le danger de perdre des ressources humaines hautement qualifiées au profit de concurrents de plus grande taille disposés à offrir des conditions financières plus alléchantes. Cette situation de concurrence, avec plusieurs autres facteurs de contingence, explique en partie le recours croissant à des formes plus complexes d'incitation et d'intéressement, bien que ces méthodes demeurent facultatives en France (pour les PE de moins de 100 salariés) et dans la plupart des pays industrialisés. Par ailleurs, il en va tout autrement pour l'évaluation du rendement, qui s'avère nettement moins formalisée dans les PME. Avec la planification des ressources humaines et l'analyse des emplois, elle constitue la pratique la moins structurée et la plus galvaudée par les propriétaires-dirigeants qui effectuent bien souvent eux-mêmes une appréciation paternaliste des performances de leurs employés. Comme pour la rémunération, le niveau de formalisation de l'évaluation du rendement est influencé par la taille organisationnelle ainsi que par la syndicalisation. C'est ainsi que les politiques écrites d'évaluation du rendement sont trois fois plus fréquentes dans les organisations de 150 employés, comparativement aux TPE de moins de 25 personnes. Les critères d'évaluation retenus concernent habituellement la personne et ses résultats plutôt que des comportements professionnels, difficiles à définir et à observer de façon continue. Bref, les pratiques de conservation des ressources humaines, bien que généralement peu formalisées, existent à des degrés fort variables, faisant ainsi encore ressortir le caractère fortement hétérogène des PME, limitant dès lors toute généralisation concernant les pratiques de GRH qu'on y retrouve.

\section{ABSTRACT}

Second of a trilogy dedicated to human resource management (HRM) in small businesses (SMEs), this paper summarizes a study that describes and comments the major HRM maintenance activities in small and medium-sized firms. Much of the available international literature has been reviewed and analyzed, based on empirical data obtained from several European and NorthAmerican surveys. A first global trend shows that, overall, compensation and fringe benefits are the most formalized HRM activities. Between 45 and $80 \%$ of SMEs formalize these practices through policies and procedures, these figures increasing with organizational size and unionization level. In fact, SME owners and managers pay particular attention to these HRM activities as they constantly face losing their highly qualified resources to larger competitors, and are more likely to offer attractive compensation packages. These competitive constraints, along with other contingency factors, partly explain the increasing use of more sophisticated incitative and involvement packages, even if these methods remain optional in France (for SMEs smaller than 100 employees) and in most other western countries.

On the other hand, the situation totally differs for performance appraisal, which is substantially less formalized in SMEs. With HR planning and job analysis, this appraisal is the least structured and the most misapplied by owners and managers, who often personally evaluate their employees' performance in a paternalist manner. As for compensation, the formalization level of performance 
appraisal varies with organizational size and unionization. In fact, evaluation policies are three times less frequent in firms with less than 150 employees, compared to small firms having less than 25 persons. Evaluation criteria usually refer to the individual employee and to his results, rather than to his professional behaviors, and are thus harder to define and to observe on a continuous basis. Finally, HR maintenance activities, whilst generally less formalized, occur in SMEs at various levels, again emphasizing the strong heterogeneous character of small and medium-sized firms, thus limiting any kind of generalization related to their HRM practices.

\section{RESUMEN}

Segundo de una trilogía dedicada a la gestión de recursos humanos, este artículo resume un trabajo orientado a describir y comentar los principales procedimientos de conservación de los recursos humanos en el contexto de las PyMES. Con este fín, ha sido analizada la experiencia internacional, mediante documentación empírica que aporta resultados sobre el terreno en diversos paises de Europa y América del Norte. Una primera constatación indica que en conjunto, la remuneración y los beneficios sociales, son en gestión de recursos humanos, las actividades más formalizadas por las politicas y procedimientos, ya que entre un 45 y un $80 \%$ de las PyMES formalizan estas prácticas, aumentando los porcentajes con el tamaño de la organización y el nivel de sindicalización. Los propietarios-directivos de las PYMES, otorgan en efecto una atención particular a estas actividades de gestión de recursos humanos, ya que tienen que hacer continuamente frente al siguiente problema: el peligro de perder recursos humanos altamente cualificados, frente a la competencia de mayor tamaño capaz de ofrecer unas condiciones financieras más atrayentes. Esta situación de competencia junto a otros factores limitantes, explica en parte el recurso creciente a formas más complejas de incitación y motivación, aunque estos métodos siguen siendo optativos en Francia (para las PyMES de menos de 100 asalariados) y en la mayor parte de los países industrializados.

Sin embargo, ocurre lo contrario con la evaluación del rendimiento que se muestra mucho menos formalizada en las PyMES. Junto a la planificación de recursos humanos y el análisis de cargos, constituye la práctica menos estructurada y la peor utilizada por los propietarios-directivos, que efectuan a menudo una evaluación paternalista del rendimiento de sus empleados. Como para la remuneración, el nivel de formalización de la evaluación del rendimiento, depende del tamaño de la organización y de la sindicalización. Así es como las prácticas escritas de evaluación del rendimiento, son tres veces más frecuentes en organizaciones de 150 empleados, comparativamente a las PyMES de menos de 25 personas. Los criterios de evaluación retenidos, se refieren habitualmente a la persona y sus resultados, más que a comprotamientos profesionales difíciles de definir y observar de forma continuada. En resumen, las prácticas de conservación de recursos humanos, a pesar de estar generalmente poco formalizadas, existen en grados muy variados, haciendo resaltar todavía más el caracter fuertemente heterogéneo de las PyMES, limitando por tanto toda generalización relativa a las prácticas de gestión de recursos humanos que en ellas se observa. 


\section{Introduction}

Il est bien connu qu'un des principaux défis des dirigeants de PME consiste à conserver leurs ressources humaines, particulièrement celles détenant le plus de qualifications. Ce problème, qui semble s'appliquer dans plusieurs pays industrialisés, $s^{\prime}$ explique en partie ${ }^{1}$ par la concurrence au niveau de la demande de certains types de main-d'œuvre, les PME ayant souvent de la difficulté à offrir des conditions financières aussi alléchantes que leurs rivales plus grandes et plus riches. C'est ainsi que de nombreux dirigeants de PME déplorent la perte de certains de leurs employés ayant acquis une expertise spécifique fondamentale au bon fonctionnement de leur entreprise. En fait, ce développement d'expertise explique parfois partiellement une certaine forme de piraterie ou de recrutement agressif des grandes entreprises auprès de certains employés de PME. Face à une telle situation, il semble nécessaire de développer des pratiques de rémunération directe et indirecte susceptibles d'assurer une certaine stabilité de main-d'œuvre dans les PME.

Ces pratiques de GRH en PME font l'objet du présent article. Celui-ci aborde également l'évaluation du rendement (appréciation du personnel), en plus de la rémunération directe et des avantages sociaux (rémunération indirecte). Selon une certaine typologie d'inspiration nord-américaine, ces pratiques de GRH peuvent être englobées sous le vocable de «conservation » des ressources humaines puisqu'elles s'adressent à des employés déjà en place. Il s'agit donc d'un second bloc de pratiques de GRH, qui suit chronologiquement un premier ensemble de pratiques visant à acquérir des ressources humaines dans une organisation ${ }^{2}$. De même, le présent article incorpore les concepts de contingence et de formalisation des pratiques de GRH en PME. Il convient aussi de rappeler que ce concept de formalisation n'est associé, à nos yeux, à aucune désirabilité sociale ou administrative en contexte de PME. Il faut plutôt considérer la formalisation comme une façon d'opérationnaliser la mesure d'une variable dépendante, en l'occurrence les pratiques de GRH en PME, et ainsi de com-

1. En partie, parce qu'il demeure possible d'expliquer les modes de gestion des organisations en s'appuyant sur un nombre élevé de facteurs de contingence, qu'il soit question de taille organisationnelle, de présence syndicale, de disponibilité des ressources, de contraintes politico-légales, de type de stratégie, de domaine d'activité, de vision entrepreneuriale ou de niveau de sophistication technologique. Cet ensemble conceptuel a d'ailleurs fait l'objet de communications au cours de l'automne 1993 (Fabi, Garand et Pettersen, 1993).

2. Pour tous ces rappels à des énoncés antérieurs, référer à Fabi et Garand / Garand / Garand et Fabi en bibliographie. 
parer plus rigoureusement le niveau de développement de ces pratiques dans l'ensemble des organisations, quelle que soit la taille organisationnelle ou le facteur de contingence retenu pour l'analyse (Fabi, Garand et Pettersen, 1993).

Dans un premier temps, cet article présente quelques considérations méthodologiques relatives aux études empiriques retenues dans le cadre de notre synthèse de documentation sur la GRH en PME (Garand, 1993). Dans un deuxième temps, nous abordons successivement les diverses pratiques de conservation des ressources humaines proprement dites: la rémunération directe, les avantages sociaux, la rémunération des cadres en PME, les écarts de rémunération entre les PME et les grandes entreprises (GE) puis, finalement, l'évaluation du rendement.

\section{Considérations méthodologiques}

Comme nous l'avons précisé antérieurement, cette étude s'appuie sur une démarche exploratoire impliquant des outils de recherche documentaire, d'analyse thématique et de contenu. Réalisée à partir de quatre critères de sélection déjà cités, cette synthèse des pratiques de conservation des RH en PME porte plus particulièrement sur 54 études empiriques regroupant plus de 9000 entreprises de petite et moyenne dimensions.

Dans le premier tableau présenté la page suivante, on constate la croissance soutenue de l'intérêt accordé aux PME, grâce au regroupement de certaines données décrivant les 54 études empiriques portant sur la conservation des RH en PME. On y note une forte concentration des enquêtes depuis 1980 ( $78 \%$ pour l'ensemble des pays, les plus anciennes provenant uniquement des États-Unis), le statut professoral des chercheurs (84\%) et la disparité des médias utilisés pour la diffusion de ces recherches. Il convient aussi de préciser la provenance internationale des travaux, dont l'importante proportion d'études menées aux États-Unis (46\%), ainsi que la diffusion accrue dans des périodiques de niveau académique ( $58 \%$ ) par rapport à $15 \%$ seulement pour les autres pays. Les travaux portant sur les pratiques de rémunération et d'évaluation du rendement semblent avoir davantage de finalités professionnelles et privées, plutôt qu'essentiellement académiques. En outre, les échantillons des études traitant de conservation des RH paraissent plus consistants (en nombre d'entreprises) par rapport aux autres pratiques de GRH (Garand, 1993 ; Fabi et Garand, 1993). Enfin, les principales études proviennent surtout d'Amérique du Nord, l'Europe ne contribuant à l'analyse de ces pratiques que de manière indirecte, au sein de recherches plus globales. 
TABlEAU 1

Compilation globale des études analysées : conservation des ressources humaines

(fréquence et pourcentage du nombre total d'études, pour chaque rubrique)

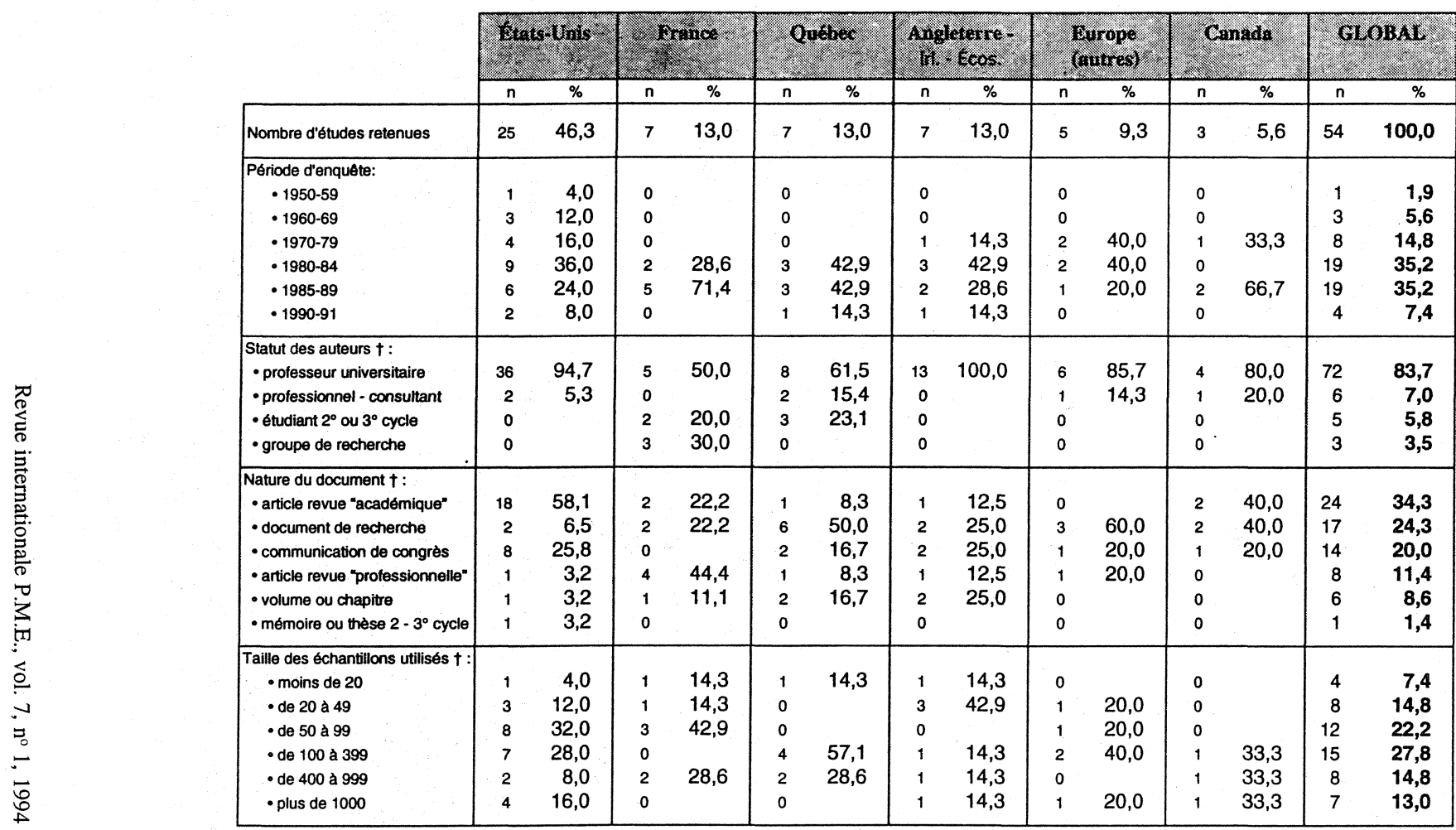

$\dagger:$ le nombre total des " $n$ " peut différer du nombre d'études retenues pour chaque pays correspondant 
Avant d'aborder l'analyse des pratiques réellement appliquées en PME, il faut aussi rappeler les contraintes imposées par l'importante hétérogénéité des travaux réalisés dans ce domaine: périodes d'enquête, populations, échantillons, méthodologies, interprétations, publications, etc.; ces facteurs compliquant la description et l'analyse des pratiques de GRH en PME, ils rendent parfois impossible toute comparaison des résultats.

\subsection{Analyse méthodologique des études empiriques}

L'analyse méthodologique des études empiriques traitant de conservation des RH en PME corrobore substantiellement le préjugé observé lors de congrès internationaux réunissant des chercheurs européens et nord-américains: les méthodes d'enquête et l'utilisation des études empiriques en GRH diffèrent d'un continent à l'autre. Ainsi, l'enquête sur le terrain détient une importance capitale en Amérique du Nord, comparativement à l'Europe où les travaux théoriques et conceptuels en GRH reçoivent davantage d'attention et souffrent souvent d'un manque de données empiriques fondamentales, particulièrement en matière de rémunération (tableau 2).

Primo, $83 \%$ des études utilisent un questionnaire d'enquête (inférieur de $7 \%$ aux pratiques d'acquisition), les proportions variant surtout en fonction des pays, mais aussi du recours aux bases de données informatisées pour la rémunération (particulièrement aux États-Unis) et aux panels pour l'évaluation du rendement (plus rares). Secundo, 29\% des travaux analysés mentionnent explicitement l'utilisation d'un prétest avant de passer à l'enquête proprement dite $(3 \%+<$ qu'acquisition), et seulement $18 \%$ effectuent un suivi après l'enquête $(8 \%+<)^{3}$. On remarque aussi le peu d'information publiée à ce sujet, comme nous l'avions souligné auparavant.

Tertio, on note pour les pratiques de conservation des $\mathrm{RH}$ une différence moins prononcée (par rapport aux pratiques d'acquisition) dans les modalités d'administration des questionnaires entre l'Europe et l'Amérique du Nord. Ainsi, les chercheurs européens se servent plus régulièrement des enquêtes postales sans entrevue $(53 \%)$ et recourent moins souvent aux entrevues directes $(47 \%)$, tandis que les Nord-Américains utilisent abondamment les envois postaux $(73 \%)$ sans effectuer d'entrevue $(69 \%)$, seulement $31 \%$ des questionnaires y étant complétés lors d'entrevues en personne. Incidemment, les études québé-

3. Toutefois, la majorité des études n'abordent pas cette question; on ne peut donc prétendre avec certitude qu'elles ne les utilisent pas. 
Tableau 2

Aperçu global des méthodologies d'enquête utilisées : conservation des ressources humaines (fréquence et pourcentage; pour chaque rubrique; par pays)

\begin{tabular}{|c|c|c|c|c|c|c|c|c|c|c|c|c|c|c|}
\hline & \multicolumn{2}{|c|}{ Ginno Unisi } & \multicolumn{2}{|c|}{ Trance } & \multicolumn{2}{|c|}{ Oubber } & \multicolumn{2}{|c|}{$\begin{array}{l}\text { Angleterric } \\
\text { In:- Beos: }\end{array}$} & \multicolumn{2}{|c|}{$\begin{array}{l}\text { Aurope } \\
8 \text { ocons }\end{array}$} & \multicolumn{2}{|c|}{ Cinadia } & \multicolumn{2}{|c|}{ GLonat } \\
\hline & $n$ & $\%$ & $n$ & $\%$ & $n$ & $\%$ & $n$ & $\%$ & $n$ & $\%$ & $n$ & $\%$ & $n$ & $\%$ \\
\hline 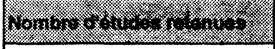 & 25 & 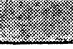 & 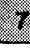 & 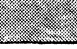 & 7 & . & ? & 20 & 5 & 20 & 2 & 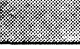 & 54 & \\
\hline Utilisation d'un questionnaire $†$ & 18 & 72,0 & 7 & 100,0 & 6 & 85,7 & 7 & 100,0 & 5 & 100,0 & 2 & 66,7 & 45 & 83,3 \\
\hline $\begin{array}{l}\text { Administration du questionnaire } \\
\text { - avec pré-test } \\
\text { - envol postal } \\
\text { - aucune entrevue } \\
\text { - entrevue en personne } \\
\text { - suivi post-entrevue }\end{array}$ & $\begin{array}{r}4 \\
12 \\
12 \\
6 \\
6\end{array}$ & $\begin{array}{l}22,2 \\
66,7 \\
66,7 \\
33,3 \\
33,3\end{array}$ & $\begin{array}{l}4 \\
3 \\
3 \\
4 \\
1\end{array}$ & $\begin{array}{l}57,1 \\
42,9 \\
42,9 \\
57,1 \\
14,3\end{array}$ & $\begin{array}{l}3 \\
5 \\
4 \\
2 \\
0\end{array}$ & $\begin{array}{l}50,0 \\
83,3 \\
66,7 \\
33,3\end{array}$ & $\begin{array}{l}2 \\
3 \\
3 \\
4 \\
1\end{array}$ & $\begin{array}{l}28,6 \\
42,9 \\
42,9 \\
57,1 \\
14,3\end{array}$ & $\begin{array}{l}0 \\
4 \\
4 \\
1 \\
0\end{array}$ & $\begin{array}{l}80,0 \\
80,0 \\
20,0\end{array}$ & $\begin{array}{l}0 \\
2 \\
2 \\
0 \\
0\end{array}$ & $\begin{array}{l}100,0 \\
100,0\end{array}$ & $\begin{array}{r}13 \\
29 \\
28 \\
17 \\
8\end{array}$ & $\begin{array}{l}28,9 \\
64,4 \\
62,2 \\
37,8 \\
17,8\end{array}$ \\
\hline $\begin{array}{l}\text { Autres modes d'enquete } \dagger \text { : } \\
\text { - base de données } \\
\text { - panel } \\
\text { - étude de cas, monographie } \\
\text { - autre }\end{array}$ & $\begin{array}{l}7 \\
0 \\
0 \\
0\end{array}$ & 28,0 & $\begin{array}{l}0 \\
3 \\
1 \\
0\end{array}$ & $\begin{array}{l}42,9 \\
14,3\end{array}$ & $\begin{array}{l}1 \\
0 \\
0 \\
1\end{array}$ & 14,3 & $\begin{array}{l}0 \\
0 \\
1 \\
0\end{array}$ & 14,3 & $\begin{array}{l}1 \\
0 \\
0 \\
0\end{array}$ & 20,0 & $\begin{array}{l}1 \\
0 \\
0 \\
0\end{array}$ & 33,3 & $\begin{array}{r}10 \\
3 \\
2 \\
1\end{array}$ & $\begin{array}{r}18,5 \\
5,6 \\
3,7 \\
1,9\end{array}$ \\
\hline Études longitudinales $†$ & 1 & 4,0 & 0 & & 0 & & 1 & 14,3 & 0 & & 0 & & 2 & 3,7 \\
\hline $\begin{array}{l}\text { Statut des intervieweurs }{ }^{\circ} \text { : } \\
\text { - professeurs universitaires } \\
\text { - étudiants universitaires } \\
\text { - professionnels }\end{array}$ & $\begin{array}{l}2 \\
3 \\
1\end{array}$ & : & $\begin{array}{l}3 \\
3 \\
0\end{array}$ & : & $\begin{array}{l}1 \\
1 \\
1\end{array}$ & : & $\begin{array}{l}2 \\
1 \\
2\end{array}$ & : & $\begin{array}{l}1 \\
0 \\
0\end{array}$ & • & $\begin{array}{l}0 \\
0 \\
0\end{array}$ & & $\begin{array}{l}9 \\
8 \\
4\end{array}$ & $\begin{array}{l}42,9 \\
38,1 \\
19,0\end{array}$ \\
\hline $\begin{array}{l}\text { Enquétes menées auprès de }{ }^{\circ}: \\
\text { - propriétaires-dirigeants } \\
\text { - cadres supérieurs } \\
\text { - responsables du personnel } \\
\text { - employés }\end{array}$ & $\begin{array}{r}17 \\
12 \\
6 \\
4\end{array}$ & $\begin{array}{l}43,6 \\
30,8 \\
15,4 \\
10,3\end{array}$ & $\begin{array}{l}4 \\
5 \\
6 \\
1\end{array}$ & $\begin{array}{r}25,0 \\
31,3 \\
37,5 \\
6,3\end{array}$ & $\begin{array}{l}3 \\
1 \\
5 \\
0\end{array}$ & $\begin{array}{l}33,3 \\
11,1 \\
55,6\end{array}$ & $\begin{array}{l}5 \\
1 \\
0 \\
3\end{array}$ & $\begin{array}{l}55,6 \\
11,1 \\
33,3\end{array}$ & $\begin{array}{l}4 \\
1 \\
0 \\
0\end{array}$ & $\begin{array}{l}80,0 \\
20,0\end{array}$ & $\begin{array}{l}0 \\
0 \\
2 \\
0\end{array}$ & 100,0 & $\begin{array}{r}33 \\
20 \\
19 \\
8\end{array}$ & $\begin{array}{l}41,3 \\
25,0 \\
23,8 \\
10,0\end{array}$ \\
\hline
\end{tabular}

N.B. : les études empiriques retenues ne fournissent pas toutes les informations relatives à leur méthodologie d'enquête

$\dagger$ : en fonction du nombre total d'études retenues pour chaque pays $\left(1^{\circ}\right.$ ligne $) \quad 0^{\circ}:$ selon le total des réponses à cette rubrique, par pays

- : en fonction du nombre total d'études utilisant un questionnaire, par pays $\left(2^{\circ}\right.$ ligne $)$ 
coises recourent à des méthodes fort similaires à celles utilisées dans les études américaines ou canadiennes, ce qui n'est pas le cas pour les études traitant d'acquisition des RH. Cependant, nous devons signaler une fois de plus le caractère inapproprié des enquêtes par questionnaires envoyés par la poste aux PME. Il serait plutôt souhaitable d'utiliser les entrevues directes afin d'assurer la participation concrète de l'entrepreneur et d'éviter d'importants biais méthodologiques liés aux répondants.

Parmi les travaux portant sur la conservation des RH en PME, on ne retrouve que deux études longitudinales, l'une américaine et l'autre britannique, et encore, elles ne s'étendent que sur une période d'un an. Le tableau 2 révèle aussi que les intervieweurs sont davantage des professeurs (43\%) ou des étudiants universitaires $(38 \%)$ et que les enquêtes s'adressent largement aux propriétaires-dirigeants ( $41 \%$ ), aux cadres supérieurs $(25 \%)$ et aux responsables des RH ( $24 \%$ ), les autres employés occupant une position bien secondaire (10\%). Il semble, en outre, pertinent de signaler l'absence complète d'entrevues téléphoniques dans ces études empiriques, le recours au téléphone étant à nouveau limité aux premières approches avec l'entreprise, à la sélection des participants, à la prise de rendez-vous, au rappel des questionnaires et à la correction des réponses mal complétées par les répondants ou les enquêteurs.

Finalement, l'analyse méthodologique des études retenues a fait ressortir les mêmes faiblesses observées en matière d'acquisition des $\mathrm{RH}$, liées pour la plupart aux impondérables de la recherche empirique en contexte de PME:

1. les schèmes expérimentaux demeurent généralement peu complexes et souvent inexistants ;

2. la taille et la répartition des échantillons par catégories de taille laisse entrevoir de nombreuses lacunes;

3. les études empiriques couvrent trop souvent plusieurs secteurs d'activité non comparables;

4. l'enquête par questionnaires envoyés par la poste s'avère inappropriée en PME;

5. la difficulté, voire l'impossibilité, de recourir à des méthodes d'analyse statistique sophistiquées lorsqu'on traite des données provenant d'échantillons de PME, en se limitant plutôt à dégager des tendances lourdes basées sur des statistiques descriptives et des tests de comparaison. 


\subsection{Critique des échantillons de certaines études}

Bien que nous ayons déjà formulé certaines critiques auparavant ${ }^{4}$, quelques études ont exigé une attention particulière à la suite de l'observation de biais dans leurs échantillons ou méthodes de recherche. Même si chaque étude empirique peut laisser place à une saine critique méthodologique, nous n'ajoutons ici que les études non commentées précédemment, afin de modérer l'interprétation des résultats présentés aux sections suivantes.

Tout d'abord, les enquêtes professionnelles de Le Louarn et Thériault (1984) et Thériault et Le Louarn (1984) s'appuient sur des échantillons comprenant plusieurs filiales de compagnies américaines ainsi que des hôpitaux, des collèges, des municipalités et des GE aux noms fort connus, qui y sont considérés PME par leur nombre d'employés, sur une base d'établissement. On observe les mêmes biais dans Thériault (1986), mais ils sont cette fois reconnus et dûment explicités : $35 \%$ des entreprises ont moins de 100 employés, ce qui constitue une nette sous-représentation par rapport au $53 \%$ d'entreprises québécoises de même taille; et plus de $60 \%$ ont moins de 250 employés. Les autres catégories $(250-499,500-999,1000+)$ sont toutes surreprésentées à peu près également de $5 \%$ chacune : $15-10 \%, 10-5 \%, 11-3 \%$. Un tel biais en faveur des plus grandes entreprises provient du recours à des banques de données contenant très peu de réelles PME : Canadian Key Business Directory et Dun \& Bradstreet (Canada). L'enquête s'appuie donc sur un échantillon très représentatif de ME et GE du Québec et l'auteur souligne qu'il n'apparaît pas important que les PE (moins de 100 employés) soient sous-représentées, parce que les entreprises de plus de 100 salariés présentent un plus grand intérêt en matière de politiques de rémunération. Comme pour les études de 1981 et 1984, celle-ci laisse donc entrevoir une proportion inférieure de PME «réelles», et on y retrouve aussi des répondants des secteurs public et parapublic. L'interprétation de ces résultats doit alors se faire avec quelques réserves.

Par ailleurs, il faut absolument souligner l'excellente qualité méthodologique des travaux menés en Angleterre par Daniel et Millward (1985), aux États-Unis par Hornsby et Kuratko (1990) et en France par Bayad et Herrmann (1992), qui fournissent une description complète de leur méthodologie et de leur échantillon, en plus de présenter des résultats parfaitement bien répartis par catégories de taille organisationnelle, en fonction des pratiques de GRH réellement appliquées en contexte de PME. Bien entendu, plusieurs autres études

4. Le lecteur doit se référer notamment dans Garand (1993) aux critiques des études suivantes : Baker (1955) ; Paumier et Gouadain (1984) ; Mahé de Boislandelle $(1985,1988,1990)$; Catherine (1990) ; Mealiea et Lee (1980) ; Thacker et Cattaneo (1987) ; Audet et al. (1987) ; McEvoy (1983, 1984); Benoit et Rousseau (1990). 
empiriques comportent des lacunes méthodologiques ou des qualités particulières, mais nous avons volontairement limité cette critique aux biais les plus manifestes, principalement pour les travaux qui fournissent une quantité considérable de données.

\section{Conservation des ressources humaines en PME: rémunération directe}

$\mathrm{Au}$ cours de la dernière décennie, plusieurs auteurs ont observé que les PME consacrent davantage d'effort aux pratiques de rémunération et d'avantages sociaux, pour lesquelles la concurrence impose des contraintes inéluctables en offrant aux individus plus qualifiés des plans de rémunération plus attrayants. Dans un contexte économique en rapide transformation, tant à l'échelle internationale que dans la répartition des activités secondaires et tertiaires dans le tissu économique, les PME n'ont d'autre choix que de s'aligner sur les pratiques des GE. Cependant, ces efforts ne s'étendent habituellement pas aux activités d'évaluation du rendement, lesquelles demeurent fortement biaisées et limitées en PME aux seules finalités salariales et disciplinaires, au lieu de servir des objectifs de conservation et de développement des ressources humaines. L'évaluation du rendement y semble d'ailleurs confondue dans bien des cas avec l'analyse des emplois, l'évaluation des postes et l'appréciation paternaliste du travail accompli par le personnel.

Cette seconde partie sera donc consacrée à la synthèse des diverses dimensions sous-jacentes à la rémunération et à l'évaluation du rendement en contexte de PME. On y traite successivement de la rémunération directe, des avantages sociaux (rémunération indirecte), de la rémunération des cadres en PME, des écarts de rémunération entre les PME et les GE puis, finalement, des divers aspects de l'évaluation du rendement (appréciation du personnel).

Après avoir recruté son personnel, toute organisation doit rémunérer adéquatement les ressources humaines qu'elle vient d'acquérir sur un marché du travail de plus en plus concurrentiel. Qu'elle soit petite ou grande, l'entreprise n'a d'autre choix que de se conformer aux normes prévalant dans son pays, sa région et son secteur d'activités ${ }^{5}$ En fait, contrairement à quelques pratiques

5. Il convient de rappeler que la formalisation des pratiques de GRH découle fréquemment des obligations imposées par les législations sociales en vigueur dans certains pays, tout particulièrement pour la rémunération. Cependant, bien que cette formalisation puisse parfois influencer le maintien des salariés dans l'entreprise, elle ne peut en aucun cas et ne doit pas être considérée comme une condition sine qua non pouvant être généralisée à l'ensemble des PME. 
de GRH moins utilisées en PME, la rémunération ne souffre d'aucune exception : tout employeur doit rémunérer son personnel en échange d'une prestation de travail. D'autre part, plusieurs aspects de la rémunération ont été traditionnellement appliqués en PME sans autre forme d'adaptation, et certains ajustements pourraient améliorer la situation dans de nombreux cas.

Globalement, les PME accordent à la rémunération directe une importance de premier plan afin de conserver un avantage comparatif sur leurs concurrents, d'attirer des ressources qualifiées, ou de combler des besoins insatisfaits auparavant en GE. Les perceptions des dirigeants et responsables du personnel en PME quant à l'importance relative qu'ils accorderont dans le futur à la gestion et aux ressources humaines révèlent que la rémunération et les avantages sociaux occupent une position prioritaire $\left(1^{\mathrm{re}} \mathrm{à} 3^{\mathrm{e}}\right.$ ), encore plus forte en PE (Hornsby et Kuratko, 1990). Toutefois, la PME doit tenir compte d'une contrainte majeure: son plan de rémunération doit absolument demeurer en deçà des limites financières de l'organisation. L'entreprise doit avoir la capacité réelle de payer ce qu'elle désire offrir à ses employés, et comme ces moyens sont souvent restreints, le propriétaire-dirigeant doit impérativement réaliser une analyse approfondie avant de s'engager dans des promesses sans lendemain.

Par ailleurs, Fay et Maurer (1986) signalent l'importance de maintenir une équité à toute épreuve dans les questions de rémunération. Tous les employés comparent leur rémunération avec leurs collègues ou amis, dans des postes similaires ou différents, à l'intérieur comme à l'extérieur de l'organisation. Ces comparaisons déterminent leur perception d'équité quant à leur situation particulière: équité externe, comparaison avec des postes similaires dans d'autres firmes; équité interne, comparaison avec d'autres postes de l'organisation; équité individuelle, comparaison entre employés de mêmes postes dans la firme.

Malheureusement, dans les premières années d'activité d'une PME, les préoccupations relatives au personnel et à la rémunération ne semblent pas du tout concerner les propriétaires-dirigeants, du moins à ce stade de développement de l'entreprise (Dolan et al., 1990). Cette négligence entraîne fréquemment le départ de ressources humaines de grande qualité dès que la PME commence à percer sur le marché : les concurrents attirent alors facilement ces individus mal rémunérés (Neiswander et al., 1987; Verser, 1987).

En France, l'ensemble des questions de rémunération et d'avantages sociaux filtre très peu hors des PME. Celles-ci sont peu syndiquées, et parmi les revendications majeures des syndicats présents en PME, on retrouve en première place les salaires et, loin derrière, l'attribution des avantages sociaux, ce qui ne constitue pas toujours une priorité pour les mouvements ouvriers français (CÉGOS, 1987). Cependant, il semble y avoir une quasi-généralisation des primes et avantages divers offerts aux cadres et employés des PME françaises, 
bien que les PME se situent toujours en deçà des GE en matière de conditions générales de travail et de rémunération, malgré des efforts considérables (Mahé et al., 1985).

\subsection{Décisions et responsabilités de rémunération}

Peu de données existent sur les politiques de rémunération en contexte de PME. Ce domaine demeure souvent la chasse gardée du propriétaire-dirigeant, même s'il semble formaliser assez rapidement ses procédures salariales (Baker, 1955; Venet, 1988 ; Benoit et Rousseau, 1990). En effet, le pourcentage d'entreprises possédant des politiques écrites de rémunération serait plus élevé que pour d'autres pratiques de GRH: il y aurait une certaine relation avec la taille organisationnelle, particulièrement dans les entreprises syndiquées (tableau 3$)^{6}$. Bien entendu, il paraît normal que ces activités soient largement formalisées en présence d'un syndicat, ces revendications étant souvent parmi les premières à être régies par une convention collective. Les formulaires utilisés en PME pour les tâches de rémunération ne sont pas nombreux, quoiqu'ils paraissent tout de même courants dans les firmes syndiquées et celles comptant plus de $150 \mathrm{em}$ ployés. Enfin, la responsabilité des activités de rémunération varie selon la nature des tâches (tableau 3) et le secteur d'activité. L'élaboration des échelles salariales et de la rémunération globale relève du propriétaire-dirigeant, celuici déléguant presque totalement la tenue des fichiers de paie et du personnel à divers subordonnés : employé en GRH, secrétaire ou autre (Little, 1986).

\subsection{Détermination des salaires}

Comme le laisse entrevoir le tableau 4, toutes les méthodes et outils de détermination des salaires paraissent moins fréquentes en PME (Mealiea et Lee, 1980): l'analyse des emplois, les échelles de salaires et les enquêtes y constituant l'essentiel des moyens utilisés. Selon Amba-Rao et Pendse (1982), les PME devraient davantage faire connaître aux employés la position compétitive favorable de leur entreprise en matière salariale (si c'est le cas...) par le biais des

6. Lecture des données statistiques présentées dans les tableaux. Les pourcentages indiquent que $\mathrm{xx} \%$ à yy \% des PME échantillonnées (résultats tirés de diverses études empiriques) possèdent ou utilisent telle ou telle méthode $\mathrm{A}, \mathrm{B}$ ou $\mathrm{C}$, généralement appliquée dans cette pratique de GRH. Les flèches indiquent une proportion $(\%)$ croissante $(\boldsymbol{x})$ ou décroissante $(\boldsymbol{\chi})$ en fonction de la taille organisationnelle (en nombre d'employés) ; la double flèche inversée $(\leftrightarrow)$ signifiant que les pourcentages ne diffèrent pas significativement selon la taille des entreprises. 
TABLEAU 3

Décisions et responsabilités de rémunération en PME

(grandes tendances observées ; données tirées de plusieurs études empiriques ; par ordre décroissant d'importance)

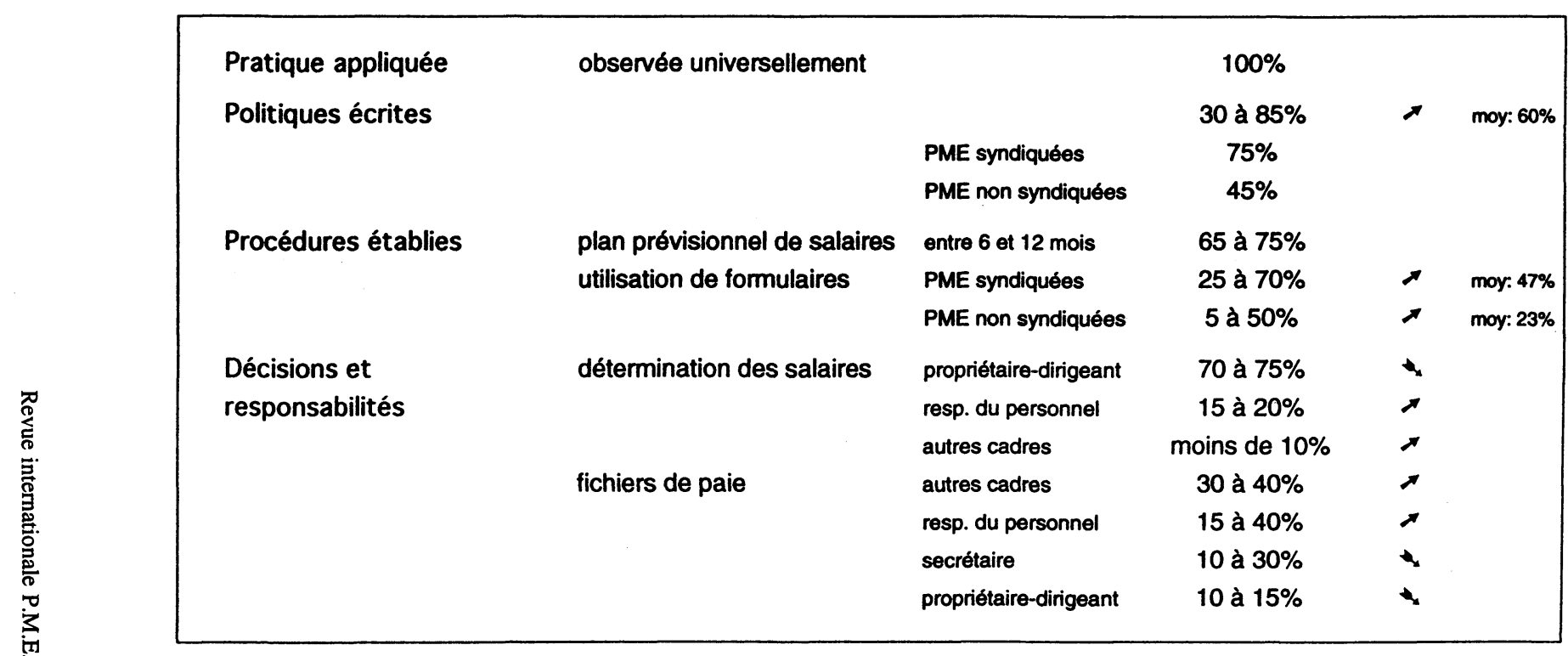


réseaux locaux d'information sur les salaires et conditions de travail (revues, centres d'emploi, annonces). De cette façon, les PME d'un secteur donné pourraient mieux harmoniser leurs conditions de rémunération et faciliter en même temps la détermination de leurs propres niveaux salariaux. Encore une fois, les méthodes formelles paraissent plus répandues dans les firmes de plus grande taille (Hornsby et Kuratko, 1990).

Ainsi, les PME déterminent leurs salaires de base à partir de critères souvent informels, tant dans les enquêtes salariales que lors de l'évaluation des emplois (tableau 4); du moins le degré de sophistication des méthodes utilisées augmente-t-il légèrement d'une catégorie à l'autre. Les entreprises de 500 employés et plus utilisent davantage les enquêtes salariales formelles que les firmes de plus petite taille. Quant aux enquêtes dirigées, les plus grands utilisateurs sont les MGE (250-499), et en évaluation d'emplois, la GE fait appel à des méthodes formelles, les moins de 250 employés, à l'évaluation informelle (Thériault, 1986). Toutefois, certaines PME utilisent une variété appréciable de méthodes, les techniques les plus courantes étant le taux du marché, l'ancienneté, les exigences du poste, l'évaluation du rendement et le salaire minimum légal.

\section{TABLEAU 4}

\section{Modes de détermination des salaires en PME}

(grandes tendances observées; données tirées de plusieurs études

empiriques ; par ordre décroissant d'importance)

ancienneté, expérience, qualification des individus

capacité de payer de l'entreprise

enquêtes salariales

connaissances, intuition de la direction

échelles salariales

aspirations du personnel

exigences de l'emploi, du poste

évaluation des emplois:

formelle

informelle

évaluation du rendement

ententes collectives

30 à $75 \%$
$70 \%$
30 à $60 \%$
$50 \%$
35 à $40 \%$
$40 \%$
$35 \%$
moins de $15 \%$
30 à $50 \%$
$20 \%$
moins de $15 \%$

\subsection{1. Évaluation des emplois}

L'évaluation des emplois à des fins de rémunération n'apparaît pas très répandue en PME (tableau 5): $18 \%$ des répondants utilisent un quelconque système d'évaluation des postes (Venet, 1988). Elle demeure rarissime en TPE, soit 
entre 3 et $20 \%$; peu fréquente en PE (15 à 46\%) et en ME (12 à $47 \%$ ); et apparaît beaucoup plus répandue dans les PME de 150 employés et plus, soit aussi largement qu'entre 27 et $60 \%$ (Hornsby et Kuratko, 1990). Les PME n'ont pas recours aux méthodes conventionnelles établies généralement en $\mathrm{GE}$, exception faite de la méthode traditionnelle basée sur le marché et de la méthode des points. Cette pratique semble très peu formalisée et pourrait même se situer hors des préoccupations des dirigeants de PME, lesquels ont plus souvent une approche réactive en rémunération. Le recours à un quelconque système d'évaluation des emplois à des fins de comparaison interne (équité) n'a été observé que chez un tiers des répondants (McEvoy, 1984). Bref, la GE fait davantage appel à des méthodes formelles, les PME semblant préférer l'évaluation informelle.

\section{TABLEAU 5}

\section{Évaluation des emplois en PME}

(grandes tendances observées; données tirées de plusieurs études empiriques ; par ordre décroissant d'importance)

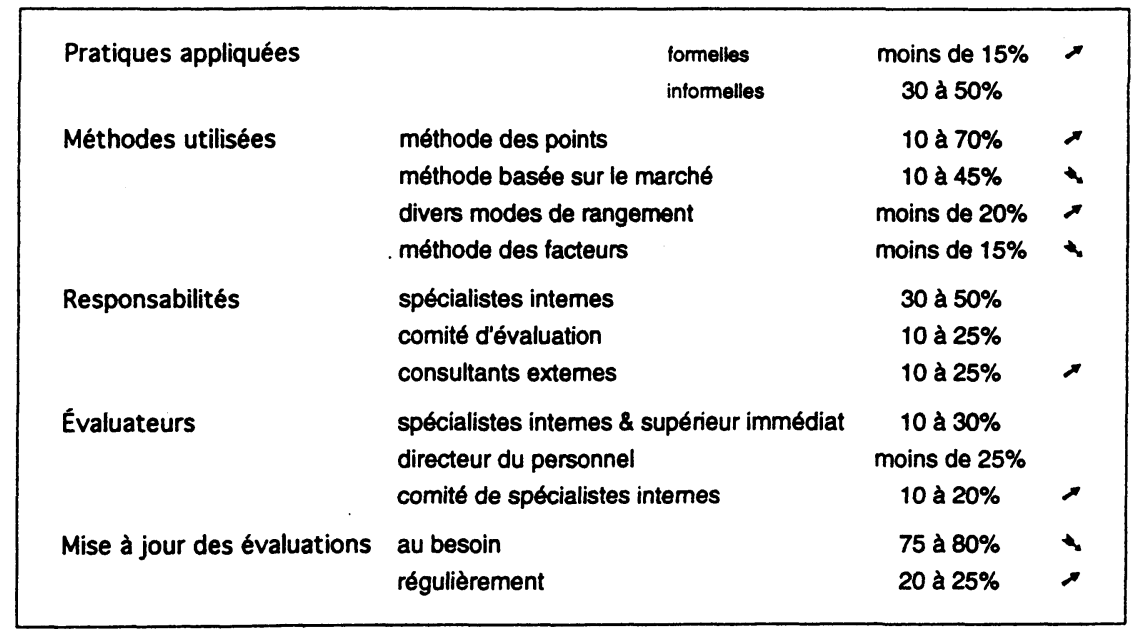

\subsubsection{Enquêtes salariales}

Cependant, le processus de détermination des salaires ne s'arrête pas à la seule évaluation des emplois. L'utilisation des enquêtes salariales s'accroît directement avec la taille organisationnelle, et ce, dans une proportion allant du simple au double (tableau 6). Près de $50 \%$ des répondants ne les utilisent pas du tout, soit qu'ils ne les connaissent pas, soit qu'ils n'en ressentent pas le besoin à cause de leur taille, soit qu'ils ne sont pas en mesure de les utiliser convenablement 
malgré leur accessibilité ou, enfin, qu'ils ne se sentent pas concernés par cette méthode tant qu'ils n'ont pas de peine à trouver leur main-d'œuvre qualifiée (Amba-Rao et Pendse, 1982). McEvoy a constaté à ce niveau la prépondérance des méthodes informelles, seulement $29 \%$ de ses répondants effectuant des enquêtes salariales formelles auprès des marchés concernés. En PE (0-99 employés), la variété des méthodes semble beaucoup moins grande, l'entrevue prédominant sur l'utilisation du courrier et les autres techniques (Thériault, 1986).

\subsection{Modes de rémunération}

En GE, des programmes et systèmes sophistiqués exigent la présence constante de spécialistes à plein temps en rémunération. Bien sûr, les PME n'ont pas besoin d'un tel support administratif, car les enquêtes salariales et la gestion de la rémunération peuvent y être réalisées à très peu de frais. Par la suite, ces services de base pourront s'étendre en fonction de la croissance de l'entreprise. De plus, quelques suggestions pourraient aider les propriétaires-dirigeants à développer un plan de rémunération comblant adéquatement les besoins des employés, tout en respectant les contraintes internes et externes ainsi que les questions d'équité individuelle. Un tel plan favoriserait la promotion d'objectifs organisationnels permettant d'attirer, de retenir et de motiver les ressources humaines sans grand risque financier: paiement de salaires compétitifs, octroi d'avantages sociaux attrayants, bonis et modes de rémunération liés au rendement des individus, services fournis par l'employeur (cafétéria, salle d'exercice) sans oublier les avantages accessibles par les regroupements d'achat (assurances, voyages, biens personnels).

\subsection{Augmentations salariales}

Le calcul des augmentations salariales peut être fait de diverses manières, mais cette opération ne doit pas nécessairement prendre la forme de hausses salariales dans leur sens étroit, car il existe d'autres possibilités liées à l'intéressement et à l'organisation du travail (Pleitner, 1982). En fait, Thériault (1986) a constaté qu'il ne semble pas y avoir de différence significative avec la taille organisationnelle dans le choix des critères d'augmentation: ceux-ci varient autour de la hausse générale des prix, la santé financière de l'entreprise et les conditions comparables sur le marché (tableau 7). Lorsqu'il est question d'augmentation individuelle de salaire, on tiendrait plus fréquemment compte des résultats de l'évaluation formelle du rendement en GE, de l'indice des prix à la consommation ainsi que des années de service dans l'entreprise de plus petite taille. La responsabilité des décisions relatives aux hausses de salaires échoit en PME 
TABleau 6

Enquêtes salariales en PME

(grandes tendances observées; données tirées de plusieurs études empiriques; par ordre décroissant d'importance)

\begin{tabular}{|c|c|c|c|c|}
\hline \multicolumn{3}{|l|}{ Pratiques appliquées } & 30 à $60 \%$ & ${ }^{\prime}$ \\
\hline \multirow[t]{6}{*}{ Enquêteurs } & \multicolumn{2}{|l|}{ service du personnel } & 55 à $85 \%$ & + \\
\hline & \multicolumn{2}{|l|}{ l'entreprise elle-même } & 45 à $60 \%$ & 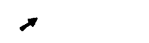 \\
\hline & \multicolumn{2}{|c|}{ organisme exteme } & 30 à $50 \%$ & 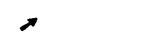 \\
\hline & \multicolumn{2}{|c|}{ service du personnel et autre intervenant } & 20 à $45 \%$ & $\lambda$ \\
\hline & \multicolumn{2}{|c|}{ enquête non commandée (sondage) } & 15 à $35 \%$ & \\
\hline & \multicolumn{2}{|c|}{ consultants externes mandatés } & 15 à $30 \%$ & $x$ \\
\hline \multirow[t]{3}{*}{ Fréquence des enquêtes } & \multicolumn{2}{|c|}{ une fois l'an } & 40 à $60 \%$ & \\
\hline & \multicolumn{2}{|l|}{ au besoin } & 20 à $45 \%$ & \\
\hline & \multicolumn{2}{|c|}{ chaque deux ou trois ans } & 10 à $20 \%$ & \\
\hline \multirow[t]{10}{*}{ Méthodes utilisées } & \multicolumn{2}{|c|}{ enquêtes dirigées spécifiquement } & 40 à $65 \%$ & + \\
\hline & \multirow[t]{2}{*}{ enquêtes générales: } & formelles & 25 à $30 \%$ & $>$ \\
\hline & & informelles & 45 à $55 \%$ & + \\
\hline & \multicolumn{2}{|c|}{ par rapport au taux moyen du marché } & 30 à $45 \%$ & 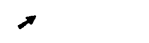 \\
\hline & \multicolumn{2}{|c|}{ par rapport au salaire minimum en vigueur } & moins de $25 \%$ & + \\
\hline & \multicolumn{2}{|c|}{ par entrevue téléphonique } & 25 à $35 \%$ & \\
\hline & \multicolumn{2}{|c|}{ par entrevue en personne } & moins de $25 \%$ & + \\
\hline & \multicolumn{2}{|c|}{ par courrier } & moins de $25 \%$ & $\pi$ \\
\hline & \multirow{2}{*}{\multicolumn{2}{|c|}{$\begin{array}{l}\text { par entrevue téléphonique et en personne } \\
\text { par courrier et entrevue en personne }\end{array}$}} & moins de $20 \%$ & + \\
\hline & & & moins de $15 \%$ & + \\
\hline \multicolumn{5}{|c|}{ Information recueillie lors des enquêtes salariales } \\
\hline \multicolumn{5}{|c|}{$\begin{array}{l}\text { Très diversifiée; il y aurait davantage d'information recueillie en grande entreprise. Cette information a principa- } \\
\text { lement trait aux aspects monétaires des salaires et avantages sociaux (minimum, maximum, moyenne, diversité), } \\
\text { et en second lieu, aux caractéristiques descriptives des firmes enquêtées. }\end{array}$} \\
\hline
\end{tabular}


à plus de $50 \%$ au propriétaire-dirigeant et au conseil d'administration (ou son équivalent); plus l'entreprise est grande, plus on accorde de pouvoir à cet égard aux supérieurs immédiats. Au contraire, ces décisions semblent davantage centralisées en PE. Enfin, $32 \%$ de l'ensemble des entreprises enquêtées possèdent un budget annuel d'augmentation au mérite, cette proportion s'accroissant avec la taille (Venet, 1988).

\section{TABLEAU 7}

Modalités des augmentations salariales en PME

(grandes tendances observées; données tirées de plusieurs études empiriques; par ordre décroissant d'importance)

Critères généraux

hausse de l'indice des prix à la consommation (I.P.C.)

50 à $60 \%$

situation financière de l'entreprise

45 à $60 \%$

augmentations accordées en général sur le marché

40 à $60 \%$

augm. accordées à du pers. semblable à l'extérieur (sondage)

augm. accordées aux autres catégories de personnel

35 à $40 \%$

15 à $35 \%$

Critères individuels

hausse de l'indice des prix à la consommation (I.P.C.)

35 à $50 \%$

évaluation formelle du rendement

30 à $40 \%$

évaluation informelle du rendement

20 à $40 \%$

niveau de salaire de la personne (compa-ratio)

20 à $30 \%$

années de service dans l'entreprise

20 à $25 \%$

années de service à l'emploi occupé

10 à $25 \%$

Responsabilité des augmentations salariales

directeur général

25 à $35 \%$

10 à $30 \%$

conseil d'administration

10 à $35 \%$

supérieur immédiat et responsable du personnel

70 à $80 \%$

date anniversaire d'embauche

20 à $30 \%$

\subsection{Régimes d'incitation et d'intéressement}

Comme le précise le tableau 8 , la rémunération ne se limite pas aux seuls salaires des cadres et employés. De plus en plus, les entreprises de toutes tailles recourent à des formes plus sophistiquées permettant de différencier, d'individualiser et de bonifier les plans de rémunération dans un marché en perpétuelle compétition. Ces nouvelles tendances ont donné naissance à divers modes 
d'incitation à la performance, d'intéressement du personnel à la gestion ou à la production, de partage des gains de l'entreprise. Dans bien des cas, ces régimes représentent plus de $30 \%$ du revenu de nombreux cadres supérieurs. Autrefois offerts principalement au personnel de vente et de production, ces incitatifs s'intègrent maintenant à la majorité des plans de rémunération des cadres de toutes catégories, et on observe depuis une décennie une proportion croissante d'employés, syndiqués ou non, qui bénéficient de tels programmes.

TABLEAU 8

Régimes d'incitation et d'intéressement offerts en PME

(grandes tendances observées; données tirées de plusieurs études empiriques; par ordre décroissant d'importance)

\begin{tabular}{|c|c|c|c|}
\hline Régimes d'incitation en PME & Efficacité relative / 5 & 25 à $45 \%$ & $\lambda$ \\
\hline Bonis individuels & 4,06 & 25 à $55 \%$ & $\varnothing$ \\
\hline Commissions & 4,15 & 30 à $40 \%$ & $\Rightarrow$ \\
\hline Primes au rendement & $\bullet$ & 30 à $40 \%$ & \\
\hline Bonis de groupe & $\bullet$ & moins de $15 \%$ & \\
\hline À la pièce & 2,64 & moins de $15 \%$ & \\
\hline Régimes d'intéressement en PME & & moins de $20 \%$ & 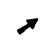 \\
\hline Partage des gains & 3,51 & 10 à $30 \%$ & $>$ \\
\hline Actionnariat & 2,49 & moins de $10 \%$ & \\
\hline En comparaison des taux horaires fixes & 3,90 & 10 à $15 \%$ & \\
\hline
\end{tabular}

D'une part, on peut constater une hausse notable du niveau de satisfaction des employés après la mise en place d'un plan de participation des employés aux bénéfices (Harrell, 1970, 1971 ; Peters, 1974; Christiansen, 1980). En outre, la participation aux bénéfices de l'entreprise et les primes de rendement développent la productivité et le sens des responsabilités des employés (Neiswander et al., 1987). D'autre part, Mahé et al. (1985) signalent que les régimes d'intéressement, les primes et avantages divers sont mis en place chez $77 \%$ des répondants, selon divers modes, même si les régimes d'intéressement aux bénéfices sont facultatifs pour les PME de moins de 100 salariés en France. Dans les entreprises comptant plus de 100 personnes, Nebenhaus (1987) et Venet (1988) ont plutôt observé des proportions voisines de $20 \%$.

De ce qui précède, nous remarquons le lien étroit prévalant en France entre les régimes d'intéressement et la participation du personnel à la gestion de 
l'entreprise, ce qui n'est pas vraiment le cas en Amérique du Nord et en Angleterre $^{7}$. En effet, ces régimes existent ici à des fins de rémunération étendue, souvent pour attirer ou conserver une main-d'œuvre qualifiée ou des cadres performants (Shaw et Lang, 1985). Par ailleurs, Thériault (1986) fournit beaucoup plus d'information sur ce sujet en PME.

1. Les régimes formels de suggestions seraient beaucoup plus fréquents en TGE, les primes les plus élevées se retrouvant surtout dans ces sociétés (jusqu'à 3000 dollars). En TPE et PE, les primes paraissent beaucoup plus rares et se situent généralement autour de 50 dollars, ces montants s'accroissant rapidement avec la taille organisationnelle. Toutefois, ils demeurent peu répandus dans toutes les catégories de taille et les échantillons trop restreints ne permettent pas de tirer de conclusions plus significatives.

2. Les régimes de partage des gains de productivité paraissent aussi rares, sans grande différence selon la taille.

3. Les régimes de bonis individuels semblent plus fréquents dans les PME de moins de 100 employés et davantage liés à la performance des personnes, en plus de quelques autres différences quant aux formules utilisées pour déterminer les bonis dans ces PE: ils sont presque essentiellement en espèces ( $90 \%$ et plus), non transférables ou différables, et fortement plafonnées en PME.

4. Les régimes de partage des profits seraient légèrement plus courants en PME et reposent fréquemment sur les profits avant impôts. De plus, les bonis individuels sont surtout basés sur le niveau hiérarchique, le statut et les salaires individuels. Le partage des profits se fait toujours à l'aide de bonis en espèces, quelle que soit la taille; ces bonis représentent habituellement moins de $10 \%$ du salaire en PME et la possibilité de différer les versements y paraît davantage obligatoire.

5. Les régimes d'intéressement à long terme laissent voir une relation directe avec la taille, ceux-ci étant rares en PME et assez répandus en TGE, presque toujours sous forme d'achat d'actions, plusieurs entreprises fournissant même une aide financière à cette fin.

7. La situation évolue toutefois progressivement depuis une dizaine d'années (Gasse et Carrier, 1992 : 121), avec des GE comme Cascades, Sico ou Tembec, et plusieurs PME : Colonial Élégance (40 salariés), Denis Cantin (130), Aliments Promar (32), Brosses Viking (120), Machineries G. Lupien (8), Meubles Laurier (150) ou Métal Rousseau (150). 
Finalement, Hornsby et Kuratko (1990) exposent clairement les différents niveaux d'utilisation et d'efficacité des modes d'incitation, d'intéressement et de partage des gains en PME (tableau 8). Les primes, les commissions et la participation aux bénéfices seraient les plus populaires, les commissions étant légèrement plus efficaces, et l'actionnariat des employés le moins utilisé et efficace, l'efficacité de ce dernier régime augmentant toutefois avec la taille organisationnelle.

\section{Avantages sociaux}

Depuis une décennie, les stratégies de rémunération des entreprises ont dû s'orienter vers des moyens indirects et plus malléables et ce, pour nombre de raisons allant des contrôles gouvernementaux visant à réduire l'inflation jusqu'aux mesures fiscales de plus en plus contraignantes pour les salariés de tous calibres. Les PME n'ont pas échappé à ce mouvement croissant vers la mise en place de programmes diversifiés: avantages de base, périphériques officiels (monétaires, en temps; immédiats ou différés), périphériques rapprochés (perquisites-perks, souvent en nature), périphériques éloignés (complémentaires et statutaires). En fait, ces avantages ont souvent pour objectif de personnaliser les conditions de rémunération du personnel qualifié afin de contrer les efforts des compétiteurs qui convoitent les mêmes individus. L'administration des avantages sociaux est graduellement devenue un secteur d'une grande complexité, tant en PE qu'en GE, et la présence d'experts paraît nettement requise pour interpréter et mettre en place les régimes complexes et détaillés d'assurance et de protection des employés. En réalité, toute organisation devrait posséder de tels régimes, mais certains programmes offerts en GE n'existent tout simplement pas en PME. Ces dernières n'ont habituellement besoin que d'un peu d'aide en ce domaine, sans devoir assumer les coûts d'un employé attitré à ces fonctions.

Globalement, une majorité d'entreprises recourent aux avantages sociaux, les modalités d'application et les limites de chaque régime variant de façon aléatoire selon la taille, avec quelques tendances significatives. Comme l'indique le tableau 9, les plus fréquents avantages demeurent les vacances, les congés et les pauses payés, les assurances vie et maladie ainsi que les régimes de retraite, ce dernier avantage étant offert par 50\% des répondants (résultat supérieur à tous les travaux antérieurs) (McEvoy, 1984). Aux États-Unis, la part de la rémunération totale dépensée en avantages sociaux s'élève à $9 \%$ dans les $\mathrm{PE}(n=79)$ et à $13,2 \%$ dans les firmes employant plus de 100 personnes $(n=70)$, la proportion moyenne étant de $11 \%(n=149)$ (Sutton, 1986), par rapport à la moyenne nationale qui s'établit à $13,5 \%(n=1154)$. La taille représente donc un facteur prépondérant qui affecte directement les avantages sociaux des employés, car on peut aisément constater les nombreuses différences prévalant 


\section{TABLEAU 9}

Avantages sociaux offerts en PME

(grandes tendances observées; données tirées de plusieurs études empiriques; par ordre décroissant d'importance)

\begin{tabular}{|c|c|c|c|c|}
\hline \multicolumn{2}{|l|}{ Politiques écrites } & \multirow{2}{*}{$\begin{array}{l}\text { PME syndiquées } \\
\text { PME non syndiquées }\end{array}$} & \multirow{2}{*}{$\begin{array}{c}\begin{array}{c}75 \text { à } 100 \% \\
30 \text { à } 80 \%\end{array} \\
30 \text { à } 55 \%\end{array}$} & \multirow{2}{*}{$\pi$} \\
\hline \multirow[t]{5}{*}{ Horaires: } & pauses payées & & & \\
\hline & pauses "nettoyage" & & 20 à $60 \%$ & $\pi$ \\
\hline & «call-in-pay» & (3-4 heures, ou plus) & 10 à $45 \%$ & $\Rightarrow$ \\
\hline & \multicolumn{2}{|c|}{ semaine de 4 jours / horaires flexibles } & moins de $15 \%$ & \\
\hline & \multicolumn{2}{|c|}{ périodes de repas payées } & moins de $10 \%$ & \\
\hline \multirow[t]{5}{*}{ Congés avec solde : } & vacances payées & & 75 à $100 \%$ & $>$ \\
\hline & congés sociaux & & 85 à 90\% & 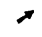 \\
\hline & congés payés & & 55 à $85 \%$ & $\Rightarrow$ \\
\hline & congés de maladie & (moins frequents en atelier) & 20 à $70 \%$ & $\lambda$ \\
\hline & congés mobiles & & 25 à $30 \%$ & $\Rightarrow$ \\
\hline \multirow[t]{9}{*}{ Assurances : } & \multicolumn{2}{|l|}{ assurance-maladie } & 70 à $100 \%$ & $>$ \\
\hline & \multicolumn{2}{|c|}{ assurance-vie } & 50 à $95 \%$ & $\Rightarrow$ \\
\hline & \multicolumn{2}{|c|}{ assurance-maladie (personnes à charge) } & 50 à $90 \%$ & $>$ \\
\hline & \multicolumn{2}{|c|}{ assurance-groupe } & 45 à $90 \%$ & $\Rightarrow$ \\
\hline & \multicolumn{2}{|c|}{ invalidité de longue durée } & 40 à $90 \%$ & $\lambda$ \\
\hline & \multicolumn{2}{|c|}{ invalidité de courte durée } & 35 à $90 \%$ & 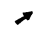 \\
\hline & \multicolumn{2}{|c|}{ assurance-vie (personnes à charge) } & 25 à $55 \%$ & $\varnothing$ \\
\hline & \multicolumn{2}{|c|}{ assurance-dentaire } & 15 à $70 \%$ & $\varnothing$ \\
\hline & \multicolumn{2}{|l|}{ soins visuels } & 5 à $40 \%$ & $\leftrightarrow$ \\
\hline Principaux avantages & \multicolumn{2}{|c|}{ stationnement } & 55 à $70 \%$ & \\
\hline \multirow[t]{8}{*}{ complémentaires : } & \multicolumn{2}{|c|}{ frais de scolarité pour les employés } & 40 à $85 \%$ & $\pi$ \\
\hline & \multicolumn{2}{|c|}{ produits ou services à prix réduits } & 40 à $60 \%$ & $\pi$ \\
\hline & \multicolumn{2}{|c|}{ frais de congrès ou conférences } & 20 à $40 \%$ & \\
\hline & \multicolumn{2}{|c|}{ congé de maternité supplémentaire } & 15 à $50 \%$ & $>$ \\
\hline & \multicolumn{2}{|c|}{ frais d'adhésion association professionnelle } & 20 à $35 \%$ & \\
\hline & \multicolumn{2}{|c|}{ plan d'épargne moy: $20 \%$} & 10 à $30 \%$ & \\
\hline & caisse d'économie & moy: $15 \%$ & moins de $30 \%$ & $\pi$ \\
\hline & \multicolumn{2}{|c|}{... et plus d'une vingtaine d'autres avantages } & moins de $20 \%$ & $\leftrightarrow$ \\
\hline Régimes de retraite : & & en TPE ( 0 - 19 employés) & 5 à $20 \%$ & \\
\hline & & en PE (20 - 99 employés) & 15 à $50 \%$ & \\
\hline & & en ME (100 - 249 employés) & 25 à $60 \%$ & \\
\hline & & en MGE ( 250 - 500 employés) & 25 à $55 \%$ & \\
\hline & & en GE (500 employés et plus) & 45 à $65 \%$ & $\pi$ \\
\hline & paie de séparation & & 5 à $20 \%$ & $>$ \\
\hline Services aux employés: & politiques écrites & & moins de $25 \%$ & $\not$ \\
\hline & rencontres sociales & & 45 à $85 \%$ & 4 \\
\hline & activités sportives & & 10 à $75 \%$ & $\Rightarrow$ \\
\hline & activités éducatives & & moins de $35 \%$ & + \\
\hline
\end{tabular}

Revue internationale P.M.E., vol. 7, nº 1, 1994 
entre les programmes d'assurance et de retraite offerts en PE et GE. Ainsi, les PME comptant moins de 100 employés semblent offrir un minimum d'avantages à leur personnel. À ce sujet, les propriétaires-dirigeants auraient intérêt à se regrouper afin de tirer profit des escomptes de quantité offerts par les sociétés d'assurance aux groupes plus nombreux (Sutton, 1986).

Aucune différence significative n'apparaît dans les régimes d'assurance vie, même si l'on note une légère relation directe avec la taille (tableau 9). En invalidité de courte durée (peu de relations significatives), les primes sont aussi assumées plus généreusement par l'employeur dans les TGE que dans les plus petites. Ces régimes sont généralement assurés, c'est-à-dire que les prestations sont versées par une compagnie d'assurance plutôt que par l'employeur. En outre, il y aurait une relation étroite entre les soins dentaires et la taille des entreprises: moins de $30 \%$ des $\mathrm{PE}$ offrent un tel régime comparativement à $76 \%$ des firmes comptant plus de 1000 employés. Quant aux soins optométriques (tableau 9), il n'y a que quelques différences non significatives. Enfin, il existe une panoplie d'avantages sociaux de toutes formes, ce qui vient confirmer l'absence de limites à l'originalité des produits et services pouvant être offerts aux employés et cadres d'une entreprise, quelle que soit sa taille et son secteur d'activité (Thériault, 1986).

En outre, on remarque que les firmes n'offrant pas d'avantages sociaux sont généralement plus jeunes, plus petites, emploient plus de main-d'œuvre à temps partiel et œuvrent dans le commerce de détail et les services (Bagby, 1987). De plus, les PME octroient un nombre restreint d'avantages sociaux à leur personnel : en moyenne, elles disposent de 2,73 avantages sociaux, l'écart type étant de 1,6, ce qui constitue un écart considérable par rapport aux GE qui fournissent à leurs employés une gamme complète de périphériques dépassant souvent la dizaine. Par ailleurs, Hornsby et Kuratko (1990) mettent en lumière une relation significative directe entre la taille organisationnelle et la complexité des avantages sociaux offerts au personnel. Cette différenciation s'exprime encore plus clairement entre PE et ME et entre secteurs d'activité. Les firmes manufacturières offrent un meilleur choix d'avantages sociaux au fur et à mesure que leur taille augmente, les firmes du commerce de détail, de la distribution en gros et des services semblant moins généreuses à cet égard.

\section{Rémunération des cadres en PME}

Depuis une décennie, les PME entrent de plus en plus en compétition directe avec les GE dans la chasse aux gestionnaires et cadres supérieurs (PDG). Le succès de ces recrutements dépend souvent de la valeur intrinsèque et extrinsèque des rémunérations offertes, et la plupart des études rapportent une 
relative diversité des plans de rémunération offerts aux chefs d'entreprises comptant moins de 500 employés. Chez ceux qui se sentent incapables de contrer les offres des GE, les motifs sont habituellement que la PE ne peut tout simplement pas générer assez d'argent pour offrir les mêmes plans; que les PE n'ont pas la possibilité de permettre autant de chances d'avancement que les GE ni de procurer le même genre de responsabilités à grande échelle, ce qui implique beaucoup d'individus et justifie un revenu supérieur. Bref, c'est le salaire qui souffre le plus souvent de ce manque de fonds, bien que certains répondants indiquent qu'ils ne peuvent concurrencer la diversité des avantages sociaux octroyés en GE. Toutefois, plusieurs affirment qu'il existe un bon nombre d'avantages pour un cadre exécutif à travailler en $\mathrm{PE}$, et ceux-ci peuvent souvent s'avérer plus attrayants que l'argent (Bacon, 1970).

Ainsi, la rémunération des cadres et hauts dirigeants de PME ne diffère pas substantiellement de celle de leurs collègues de GE, sauf peut-être pour la diversité des plans offerts et la valeur monétaire des salaires. Cependant, on reconnaît l'existence de motivations profondes particulières chez les PDG de PME, ce qui compenserait parfois largement l'écart de rémunération avec les GE (Bacon, 1970; Shaw et Lang, 1985). Il s'agit alors d'adapter les stratégies de rémunération des PME à ces attentes, tout en développant des avantages sociaux originaux et attrayants, à des coûts moindres que les régimes traditionnels des TGE.

\section{5. Écarts de rémunération entre PME et GE}

L'existence d'un préjugé populaire tenace à propos des écarts de rémunération entre PME et GE s'observe dans tous les pays depuis près d'un demi-siècle, et plusieurs auteurs signalent que les niveaux de rémunération augmentent généralement avec la taille organisationnelle, des employés occupant des postes comparables ayant plus de chance d'avoir une rémunération plus élevée en GE qu'en PE (Weiss et Landau, 1984; Barron et al., 1987; Bélanger, 1988; Evans et Leighton, 1989; Idson et Feaster, 1990; Kruse, 1992 ; Morissette, 1993). Bien sûr, on ne peut prétendre qu'il y ait ici relation de cause à effet, mais la diversité et la persistance des constatations statistiques permettent d'affirmer qu'il existe plus souvent qu'autrement un différentiel important entre les rémunérations en PE et GE. Il serait aussi plausible de croire, par exemple, que les emplois créés par les PME dans le secteur des services sont «plus pauvres » que ceux qui ont été perdus dans le déclin du secteur manufacturier. En fait, le tableau 10 révèle clairement que les salaires augmentent avec la taille, et la dimension de l'organisation a un effet plus important et statistiquement plus significatif que la taille de l'établissement (Brown et Medoff, 1989). 
Tableau 10

Écarts de rémunération entre PME et grandes entreprises

(grandes tendances observées; en pourcentage de la rémunération offerte en GE;

données provenant de 11 pays de l'OCDE; par catégorie de taille organisationnelle)

\begin{tabular}{|c|c|c|c|c|c|c|c|c|c|}
\hline & Nombre đemployés & 0 à 10. & 10 à 20. & 20 a 50 & 50 a 100 & 100 à 200 & 200 à 500 & 500 à 1000 & 1000 et + \\
\hline \multicolumn{2}{|c|}{ Écarts observés } & 40 à $58 \%$ & 55 à $75 \%$ & 57 à $81 \%$ & 72 à $93 \%$ & 74 à $88 \%$ & 80 à $97 \%$ & 90 à $100 \%$ & $100 \%$ \\
\hline \multicolumn{2}{|c|}{ Écarts moyens } & $55 \%$ & $65 \%$ & $70 \%$ & $75 \%$ & $80 \%$ & $88 \%$ & $98 \%$ & $100 \%$ \\
\hline N.B.: & \multicolumn{9}{|c|}{ Ces proportions varient substantiellement selon l'échantillon étudié } \\
\hline
\end{tabular}


Globalement, le personnel des TPE reçoit un salaire de 30 à $40 \%$ inférieur à celui des TGE (tableau 10). Ce différentiel diminue constamment, mais demeure à $25 \%$ en $\mathrm{PE}$, à $15-20 \%$ en $\mathrm{ME}$ et $10 \%$ en MGE, sans compter les variations considérables observées entre les divers pays analysés (OCDE, 1985). Ainsi, les employés des PME allemandes gagnent en moyenne moins que leurs collègues de GE, qu'ils soient cols blancs ou cols bleus, hommes ou femmes, et la hausse du revenu moyen en RFA, de 1981 à 1985, démontre très nettement l'existence d'une relation directe entre la taille et l'accroissement des écarts de rémunération entre PME et GE (Wassermann, 1989). En Espagne, Miguelez (1989) a constaté l'existence d'une relation directe entre la taille et la rémunération relative des travailleurs permanents à temps plein, selon laquelle les salaires réels sont supérieurs de 30 à $40 \%$ en GE et les avantages sociaux plus diversifiés. Il existerait aussi plusieurs différences salariales entre les catégories occupationnelles en fonction de la taille des établissements, les écarts étant en diminution constante entre PME et GE. Une telle différence se retrouve dans les pays asiatiques : au Japon, elle est de l'ordre de 30 à $45 \%$ et l'écart s'y accroît depuis quinze ans entre toutes les catégories de taille, contrairement aux pays européens et américains.

À notre grande surprise, la qualité des emplois offerts par les PME ne s'est pas améliorée de façon systématique au Québec, exception faite des «entreprises modèles» qui apparaissent à la une des médias. En effet, Laroche (1989) précise que ces emplois sont moins bien rémunérés qu'en GE et l'écart s'est sérieusement aggravé depuis la récession de 1981-1982. Cette grande disparité de rémunération entre les salariés des GE et ceux des PME, au détriment de ces derniers, expliquerait en partie les difficultés des PME à recruter du personnel qualifié et diplômé. Laroche justifie cette situation par le fait que les employés de GE ont bénéficié d'une meilleure sécurité offerte par des structures organisationnelles plus stables et par la présence organisée de syndicats même si, par ailleurs, les $\mathrm{GE}$ ont été sévèrement affectées dans leur emploi. Les ajustements draconiens de salaire suscités par la crise ont été réalisés surtout en TPE et PE; et la reprise n'a pas eu d'effets compensateurs puisque le salaire relatif dans ces entreprises a continué de baisser. Finalement, en raison d'une syndicalisation presque absente et de l'impact de cette situation sur les autres conditions de travail, on est forcé de croire que l'écart, par rapport aux GE, est encore plus élevé si l'on se refère au concept de rémunération globale (Laroche, 1989). Tous ces écarts de rémunération proviennent probablement des facteurs suivants (Beaucourt et al., 1989; Wassermann, 1989):

- les effets liés au marché du travail;

- les niveaux différents de prix à la consommation en milieux urbains et ruraux; 
- la plus grande proportion d'emplois de cols blancs dans les organisations à grande échelle, avec de meilleures rémunérations ;

- le pourcentage supérieur à la moyenne de travailleurs à temps partiel et temporaires en PME;

- la main-d'œuvre des PME est plus jeune, est composée surtout de femmes, possède moins d'ancienneté et provient davantage des milieux ruraux;

- on y retrouve moins de travailleurs qualifiés;

- le mode de rémunération diffère entre GE et PME : ces dernières ont un taux plus élevé de salariés horaires, d'où une plus grande flexibilité pour adapter leurs horaires de travail à la demande ;

- les modes de représentation des intérêts diffèrent considérablement entre PME et GE.

\section{6. Évaluation du rendement}

L'évaluation du rendement a souvent été considérée comme absente des pratiques courantes chez les propriétaires-dirigeants de PME. Les résultats semblent démontrer que son application réelle serait en relation directe avec la taille de l'entreprise (Baker, 1955; Thériault et Le Louarn, 1984) et le statut syndical, tout comme l'utilisation de formulaires d'appréciation augmenterait directement avec la taille. L'évaluation du rendement s'effectue surtout de façon informelle et paraît principalement basée sur l'observation.

Comme l'indique le tableau 11, le tiers des PME disposent de politiques écrites, un autre tiers en possèdent plutôt sous forme non écrite et $28 \%$ n'ont aucune politique officielle d'évaluation du rendement (Benoit et Rousseau, 1990). Les procédures formelles d'évaluation ne se retrouveraient que dans 30 à $50 \%$ des PME, tandis que moins de $25 \%$ des entreprises s'appuieraient uniquement sur l'observation, ces données différant largement selon le secteur d'activité (d'Amboise et Gasse, 1980). Près de 50\% des PME effectuent une évaluation périodique du rendement, celle-ci étant plus formelle (par écrit) dans seulement 20 à $25 \%$ des cas (Amba-Rao et Pendse, 1982; Venet, 1988).

Cependant, le suivi et le contrôle du personnel paraissent assurés de façon plus ou moins systématique pour renforcer la motivation du personnel et améliorer l'efficacité de la gestion (Roger, 1985). Par contre, $71 \%$ des PME américaines possèdent un système d'évaluation du rendement, la moyenne nationale étant aussi élevée que $95 \%$ (McEvoy, 1984). Cet état de fait souligne de nouveau le caractère fondamentalement hétérogène des PME, plusieurs études empiriques rapportant des données largement différenciées des tendances lourdes observées dans d'autres milieux. 


\section{TABLEAU 11}

\section{Évaluation du rendement en PME}

(grandes tendances observées; données tirées de plusieurs études empiriques; par ordre décroissant d'importance)

\begin{tabular}{|c|c|c|c|}
\hline Politiques écrites & moy: $\mathbf{3 2} \%$ & 20 à $50 \%$ & \\
\hline Procédures établies & & moins de $50 \%$ & \\
\hline \multirow[t]{3}{*}{ utilisation de formulaires } & & moins de $30 \%$ & $\pi$ \\
\hline & formelles & 15 à $50 \%$ & $\pi$ \\
\hline & informelles & moins de $25 \%$ & 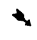 \\
\hline Objectifs de l'évaluation & \multicolumn{2}{|l|}{ Efficacité relative / 5} & \\
\hline communication et motivation & - & 55 à $75 \%$ & + \\
\hline analyse des besoins de formation et développement & 4,00 & 40 à $80 \%$ & $\pi$ \\
\hline hausse ou baisse de rémunération & 4,14 & 30 à $75 \%$ & $\lambda$ \\
\hline mouvements de personnel & 4,10 & 20 à $80 \%$ & 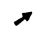 \\
\hline discipline & 3,57 & 30 à $65 \%$ & 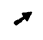 \\
\hline validation du recrutement et sélection & 3,42 & 20 à $35 \%$ & 入 \\
\hline
\end{tabular}

\subsection{Objectifs et contenu de l'évaluation}

Souvent confondus de toutes sortes de façons, les objectifs et le contenu de l'évaluation prennent différentes formes selon les entreprises (tableau 11). Bien que son but premier soit de fournir les données nécessaires à la détermination de la productivité des employés afin d'améliorer leur efficacité dans un objectif de performance organisationnelle, l'évaluation du rendement sert plus souvent qu'autrement à des fins multiples : sélection, probation, rémunération, formation, développement ou discipline (Baker, 1955; Roger, 1985). Hornsby et Kuratko (1990) confirment cette utilisation souvent biaisée de l'évaluation tout en faisant la preuve de l'existence concrète d'une relation directe avec la taille. En PE, l'évaluation du rendement se fait dans des situations très particulières (production, discipline) et tout bonnement à l'insu du principal intéressé (Holley et Wolters, 1987). Même en ME, elle apparaît peu formalisée et se pratique beaucoup plus à des fins coercitives ou de conformité. Son contenu touche les retards, l'absentéisme, la discipline, l'obéissance aux directives, la quantité et qualité du travail, etc. Dans certains cas, l'évaluation du rendement est formelle pour les cadres et quelques catégories d'employés (bureau et technique); rarement, a-t-elle pour objet le développement de l'employé et la communication, comme c'est plus souvent le cas en GE (Audet et al., 1987). 


\section{2. Évaluateurs et responsabilités de l'évaluation}

En PME, la responsabilité de l'évaluation du rendement n'incombe pratiquement jamais au responsable du personnel (tableau 12). Elle relèverait plutôt du propriétaire-dirigeant et du supérieur immédiat à mesure que l'entreprise croît en nombre d'employés, et dès qu'on dépasse les 25 employés, cette responsabilité passe du dirigeant au contremaître ou superviseur en chef, atteignant $74 \%$ en ME (Baker, 1955). Plus récemment, on a signalé le choix unanime du supérieur immédiat comme évaluateur désigné d'office dans toutes les catégories de taille (98 à 100\%), et l'importance de l'employé comme évaluateur (24\% en PME) (Thériault et Le Louarn, 1984; Roger, 1985). Par contre, certains accordent plus d'importance au responsable du personnel qui partagerait avec le propriétaire-dirigeant $25 \%$ des tâches d'évaluateur, celui-ci conservant près de $50 \%$ des responsabilités d'évaluation (Mahé et al., 1985; Little, 1986).

Tableau 12

Responsabilité et fréquence de l'évaluation en PME

(grandes tendances observées; données tirées de plusieurs études empiriques; par ordre décroissant d'importance)

\begin{tabular}{|c|c|c|c|c|}
\hline \multirow[t]{6}{*}{ Responsabilité } & Dirigeants: & propriétaire-dirigeant & $\begin{array}{l}45 \text { à } 60 \% \\
10 \text { à } 40 \%\end{array}$ & + \\
\hline & \multirow[t]{2}{*}{ Superviseurs: } & supérieur immédiat & 20 à $75 \%$ & $\pi$ \\
\hline & & superviseur en chef & 5 à $20 \%$ & \\
\hline & \multicolumn{2}{|c|}{ Responsable du personnel } & 10 à $30 \%$ & $\pi$ \\
\hline & \multicolumn{2}{|c|}{ L'employé lui-mème } & 15 à $25 \%$ & \\
\hline & \multicolumn{2}{|l|}{ Autres } & moins de $10 \%$ & \\
\hline \multirow[t]{3}{*}{ Fréquence } & \multicolumn{2}{|l|}{ annuelle } & 40 à $90 \%$ & $\pi$ \\
\hline & \multicolumn{2}{|c|}{ semi-annuelle } & 25 à $35 \%$ & $\leftrightarrow$ \\
\hline & \multicolumn{2}{|c|}{ trimestrielle; selon l'individu; quotidienne; mensuelle } & 5 à 15\% & + \\
\hline
\end{tabular}

\subsection{Fréquence de l'évaluation}

La fréquence des évaluations du rendement en PME se situerait quelque part entre six mois et un an (tableau 12). Globalement, plus la taille organisationnelle augmente, plus cette fréquence diminue, l'intervalle se prolongeant jusqu'à dix à douze mois (Baker, 1955). En outre, elle dépendrait plus souvent des individus en PME, laissant entrevoir une plus faible formalisation des processus d'évaluation (Thériault et Le Louarn, 1984). Ainsi, la fréquence la plus populaire serait annuelle (environ $45 \%$ ), puis semi-annuelle ( 25 à $30 \%$ ) et enfin trimestrielle 
(10 à $12 \%)$. Il faut noter ici que dans les «grandes» PME, l'évaluation se fait majoritairement à tous les ans, et très peu en deçà de six mois $(12 \%$ contre $28 \%$ en «petites» PME) (Audet et al., 1987; Hornsby et Kuratko, 1990).

\subsection{Méthodes et procédures d'évaluation}

On remarque aussi une similitude modérée entre les critères d'évaluation retenus en PME et ceux des plus grandes entreprises (tableau 13), à l'exception des critères de nature subjective (maturité, intégrité, stabilité émotive, ambition, intérêts de carrière) qui paraissent plus utilisés dans les firmes de moins de 249 employés et des critères liés au degré de formalisation des pratiques de GRH, qui se retrouvent davantage dans les organisations de grande dimension (planification, organisation, habiletés analytiques et de gestion, délégation, comportements, ponctualité). Pour les autres critères d'évaluation du rendement, il ne paraît pas y avoir de relation avec la taille (objectifs, connaissances, qualité, quantité de travail, etc.) (Thériault et Le Louarn, 1984).

\section{TABLEAU 13}

\section{Critères d'évaluation du rendement en PME}

(grandes tendances observées; données tirées de plusieurs études

empiriques ; par ordre décroissant d'importance)

\begin{tabular}{|c|c|c|}
\hline entre 80 et $95 \%$ : & $\begin{array}{l}\text { connaissance du travail } \\
\text { qualité du travail }\end{array}$ & objectifs de travail \\
\hline entre 70 et $85 \%$ : & $\begin{array}{l}\text { communication } \\
\text { quantité de travail }\end{array}$ & $\begin{array}{l}\text { potentiel de développ. individue } \\
\text { jugement }\end{array}$ \\
\hline entre 55 et $70 \%$ : & $\begin{array}{l}\text { intérêts de carrière } \\
\text { motivation } \\
\text { attitudes } \\
\text { organisation } \\
\text { leadership }\end{array}$ & $\begin{array}{l}\text { coopération } \\
\text { habiletés de gestion } \\
\text { planification } \\
\text { comportements }\end{array}$ \\
\hline entre 40 et $55 \%$ : & $\begin{array}{l}\text { traits / caractéristiques généraux } \\
\text { formation et développement individuel }\end{array}$ & $\begin{array}{l}\text { maturité } \\
\text { habiletés analytiques }\end{array}$ \\
\hline entre 20 et $40 \%$ : & $\begin{array}{l}\text { stabilité émotive } \\
\text { connaissance des politiques de la firme } \\
\text { ambition } \\
\text { intégrité }\end{array}$ & $\begin{array}{l}\text { contrôle de soi } \\
\text { ponctualité } \\
\text { délégation } \\
\text { évaluation du personnel }\end{array}$ \\
\hline
\end{tabular}

En outre, il existe toute une panoplie de techniques disponibles, mais la PME n'a généralement recours qu'à quatre ou cinq méthodes, variant substantiellement en fonction de la taille. Comme le précise le tableau 14, elles se limitent (par ordre décroissant d'importance) à l'établissement d'objectifs, la composition ouverte, les incidents critiques, le classement par rang, ainsi que 
les échelles graphiques et de mesure des traits, l'ensemble des autres modes d'évaluation n'étant observable qu'en GE et TGE (Thériault et Le Louarn, 1984; Thacker et Cattaneo, 1987; Hornsby et Kuratko, 1990). Cependant, la méthode la plus populaire consiste à mesurer objectivement la productivité, les GE recourant plus aux échelles de mesure des traits (McEvoy, 1984), tandis que Thacker et Cattaneo (1987) relatent que la plupart des méthodes d'évaluation n'étaient pas utilisées par leurs répondants, tout particulièrement en PME. Le recours à l'établissement d'objectifs paraît généralisé chez les cadres, mais dans une moindre mesure pour l'évaluation du personnel technique et professionnel.

\section{TABLEAU 14}

Méthodes d'évaluation du rendement utilisées en PME

(grandes tendances observées; données tirées de plusieurs études empiriques; par ordre décroissant d'importance)

\begin{tabular}{|c|c|c|c|c|c|}
\hline \multirow[t]{3}{*}{ Ensemble des méthodes } & & & & 55 à $85 \%$ & \\
\hline & efficacité relative & \multicolumn{2}{|c|}{ modérément efficace } & 50 à $65 \%$ & \\
\hline & & \multicolumn{2}{|c|}{ très efficace } & $30 \%$ & \\
\hline \multirow[t]{6}{*}{ Plus utilisées en PME } & échelles graphiqu & & $\bullet$ & 35 à $65 \%$ & \\
\hline & établissement d'o & jectifs & 4,03 & 30 à $65 \%$ & \\
\hline & classement par ra & & 3,90 & 35 à $60 \%$ & \\
\hline & composition ouve & & 3,90 & 20 à $65 \%$ & \\
\hline & incidents critiques & & $\bullet$ & 15 à $45 \%$ & \\
\hline & échelle de mesure & des traits & $\bullet$ & 25 à $35 \%$ & \\
\hline \multirow[t]{2}{*}{ Très peu utilisées en PME } & échelles pondérée & & & 15 à $20 \%$ & \\
\hline & choix forcé & & & 7 à $18 \%$ & \\
\hline (moins de 10\%) & \multicolumn{5}{|c|}{$\begin{array}{l}\text { diverses techniques de rangement; comparaison pairée; } \\
\text { séminaires; distribution imposée; WP \& R, BARS, BDS, PDA }\end{array}$} \\
\hline
\end{tabular}

Toutefois, tous ces résultats exposent les modes d'évaluation officiellement mis en place dans l'organisation par les responsables du personnel et ne permettent aucunement de savoir ce qui s'y passe réellement. De fait, dans un grand nombre d'entreprises, une large proportion du personnel semblait ignorer complètement l'existence des systèmes d'évaluation du rendement (Thacker et Cattaneo, 1987). Cette absence de méthode formelle, ou même tout simplement d'évaluation, proviendrait de l'habitude de discuter librement avec l'employé de son rendement, pratique qui semble bien ancrée en PME (70,5\%) puisqu'on observe une relation négative avec la taille. En effet, les individus y étant plus rapprochés dans tous les sens du terme, l'évaluation du rendement se déroulerait par voie de discussion informelle, serait plus fréquente et parfois plus collective (Baker, 1955). De plus, les méthodes d'évaluation du rendement varient en fonction du secteur d'activité. 


\subsection{Information et formation à l'évaluation}

L'information et la formation relative à l'évaluation du rendement ne sont pas considérées comme des activités importantes en GRH, et ceci en fonction de la fréquence des programmes et de leur contenu. Ce type d'activité revêt un degré de difficulté qui repousse peut-être les responsables du personnel : il est en effet fort difficile de porter des jugements sur la manière dont les gens s'acquittent de leurs tâches de formateur ou d'évaluateur. D'ailleurs, la formation des évaluateurs n'existe pratiquement pas en PME et les résultats diffèrent largement selon la taille. Lorsqu'elle est réalisée, l'élaboration et l'exécution des programmes de formation à l'évaluation repose presque essentiellement sur les épaules du propriétaire-dirigeant en PME (62 à $69 \%$ ), celui-ci déléguant peu à peu à des consultants (internes et externes) (Thériault et Le Louarn, 1984). En outre, l'utilisation de l'information relative à l'évaluation du rendement s'accroît avec la taille des PME (Hornsby et Kuratko, 1990).

\subsection{Efficacité de l'évaluation}

Quant à la gestion des systèmes d'évaluation, elle n'apparaît superficiellement que dans les rares PME disposant d'un système formel, tandis que la vérification de l'efficacité de l'évaluation s'effectue surtout par discussion informelle avec les intéressés, une majorité de PME se disant généralement satisfaites de leur processus d'évaluation (Thériault et Le Louarn, 1984). En effet, la majorité des PME étudiées y accordaient un degré d'efficacité parmi les plus élevés des pratiques de GRH, avec des efficacités relatives se situant entre 3,50 et 4,25 pour diverses activités d'évaluation. Il faut aussi noter au tableau 11 l'importante efficacité relative accordée aux trois premiers objectifs de l'évaluation du rendement, cotés entre 3,91 et 4,22 en TPE (4,00 à 4,14 en PE; 3,98 à 4,20 en ME) pour des taux moyens se situant entre 4,00 et 4,14 . Quant à l'efficacité des techniques d'évaluation (tableau 14), l'établissement d'objectifs paraissant légèrement plus efficace que le classement par rang et la composition ouverte (Hornsby et Kuratko, 1990).

\section{Conclusion}

La présente démarche de recension critique de la documentation spécialisée a permis de répondre principalement aux questions suivantes: Quelles sont les pratiques de conservation des RH appliquées dans les PME? Dans quelle mesure sont-elles implantées et quel est leur degré de formalisation? 
Les résultats démontrent qu'il existe réellement des pratiques de conservation des RH appliquées en PME. Bien qu'elles demeurent généralement peu formalisées, ces pratiques existent à des degrés fort variables, démontrant sans équivoque le caractère fortement hétérogène des PME, interdisant dès lors toute autre forme de généralisation en ce qui a trait aux pratiques de GRH. Concrètement, on retrouve en PME la plupart des pratiques de conservation des RH enseignées dans les traités académiques, bien que plusieurs PME n'aient recours qu'aux activités traditionnelles rattachées à la rémunération et à l'appréciation paternaliste (ou disciplinaire) du personnel. Bien entendu, la mise en œuvre de ces pratiques dépend largement du contexte légal et des contraintes organisationnelles, plusieurs aspects de la rémunération étant régis par des réglementations nationales, tant en Europe qu'en Amérique du Nord. Bien que ces normes légales ne soient pas systématiquement appliquées en PME, elles exercent une influence notable sur les pratiques réelles de conservation des RH.

En plus de ces normes légales, on observe que la taille organisationnelle semble également avoir une influence sur le degré de formalisation des pratiques de conservation des ressources humaines dans les PME. Globalement, la rémunération s'avère beaucoup plus complexe en GE et cela, même si les propriétaires-dirigeants de PME y accordent une attention particulière. À maintes reprises, des PME ont assisté au départ de ressources humaines hautement qualifiées vers des concurrents de plus grande taille disposés à offrir des conditions plus alléchantes. Concrètement, une minorité de PME non syndiquées disposent de politiques écrites en matière de rémunération et d'avantages sociaux, ces questions étant souvent du ressort exclusif du propriétaire-dirigeant, même si ce dernier semble formaliser rapidement ses procédures salariales à mesure qu'augmente la taille organisationnelle en déléguant alors presque totalement les tâches administratives à divers subordonnés. À l'opposé, le pourcentage de PME syndiquées ayant des politiques et procédures formelles en rémunération paraît très largement supérieur, cet élément constituant l'une des premières revendications des syndicats lors d'une négociation collective. Dans l'ensemble, la rémunération et les avantages sociaux représentent tout de même les activités de GRH les plus formalisées par des politiques et procédures, les pourcentages augmentant avec la taille et le niveau de syndicalisation (voir tableau 3).

En outre, on observe dans toutes les catégories de PME un recours croissant à des formes complexes d'incitation et d'intéressement, bien que ces méthodes demeurent facultatives en France (pour les PE de moins de 100 salariés) et dans la plupart des pays industrialisés. Combinés à des programmes d'avantages sociaux diversifiés et de plus en plus formalisés en PME, ces régimes constituent l'élément majeur de formalisation des pratiques de rémunération en PME, à un point tel que la présence d'experts externes paraît 
nettement requise pour mettre en place les régimes complexes et détaillés d'assurance et de protection individuelle, particulièrement dans les PME syndiquées où prévaut une convention collective.

Par ailleurs, l'évaluation du rendement s'avère nettement moins formalisée en PME. Avec la planification des ressources humaines et l'analyse des emplois, elle constitue la pratique la moins structurée et la plus galvaudée par les entrepreneurs qui effectuent bien souvent eux-mêmes une appréciation paternaliste des performances de leurs employés. En fait, les politiques écrites d'évaluation du rendement sont trois fois plus fréquentes dans les entreprises de plus de 150 employés, que dans les TPE de moins de 25 personnes. Les objectifs et le contenu de l'évaluation prennent diverses formes selon les entreprises et servent plus souvent qu'autrement à des fins multiples, ce qui reflète le faible degré de formalisation des procédures d'évaluation en PME. Il semble que cette pratique ne soit formalisée qu'en présence d'une convention collective et qu'elle repose souvent sur le seul engagement moral des propriétaires-dirigeants. Elle porte habituellement sur la personne et ses résultats plutôt que sur des comportements professionnels mal définis et souvent difficiles à observer de façon continue (Roger, 1985). Dans plusieurs PME, l'évaluation se fait même à l'insu du principal intéressé et se pratique beaucoup plus à des fins coercitives ou de conformité. Enfin, ces informations ne révèlent que les modes d'évaluation officiellement mis en place dans l'organisation par les responsables du personnel, comme nous l'avons signalé auparavant.

Plusieurs explications peuvent être apportées à cette sous-utilisation des pratiques de rémunération et d'évaluation du rendement en PME. Il faut tout d'abord considérer les coûts qu'entraînent l'élaboration, l'implantation et la gestion de tels systèmes formalisés. Ensuite, certains peuvent faire valoir l'aspect superflu de telles procédures, particulièrement dans l'appréciation du personnel, effectuée plus directement que dans les organisations de grande dimension. En revanche, l'importance de la taille rend l'accès à l'information et le contrôle plus difficiles, ce qui pourrait expliquer la formalisation des systèmes d'évaluation du rendement. Cette formalisation peut aussi découler d'un besoin, ressenti par certains propriétaires-dirigeants, d'améliorer la gestion et l'appréciation des charges sociales et des coûts, dépassant ainsi le simple souci de conserver leurs salariés. Enfin, il apparaît essentiel de rappeler le poids des revendications syndicales sur les dirigeants de PME qui, sous les pressions des mécanismes de négociation, sont souvent contraints à formaliser les informations et procédures relatives à la rémunération et à l'évaluation du rendement, afin d'appuyer sur des bases plus rationnelles et plus efficaces les données négociées.

Par ailleurs, l'analyse laisse entrevoir certaines limites dont il faut tenir compte dans l'appréciation des résultats qui en découlent. En premier lieu, cet examen de la documentation ne peut prétendre à l'exhaustivité. D'abord, parce 
que même si cette synthèse impliquait au-delà de 100 périodiques édités dans une douzaine de pays, elle se limitait tout de même à la documentation empirique publiée en langues française et anglaise dans quelques pays occidentaux. Ensuite, parce que parmi cette dernière, il y a possiblement eu des omissions involontaires devant la panoplie de publications académiques et professionnelles diffusées sur les plans local et régional. Une seconde limite réside dans l'hétérogénéité des apports méthodologiques de chaque étude empirique. En effet, on doit reconnaître la difficulté inhérente à la synthèse de résultats empiriques dont la qualité méthodologique s'avère fortement hétérogène, entraînant un risque élevé associé à la généralisation des données statistiques présentées. Par conséquent, l'élaboration de certains tableaux nous a obligés à tracer des tendances médianes exluant certains résultats marginaux qui correspondaient parfois à la situation réelle des pratiques de GRH dans plusieurs PME.

Ces considérations nous incitent à formuler certaines recommandations aux auteurs désireux de poursuivre des recherches portant sur les pratiques de conservation des RH en PME, recherches que nous souhaiterions de meilleure qualité méthodologique et conceptuelle. L'adaptation de la recherche aux besoins réels des PME en matière de GRH devrait s'appuyer sur une base d'information plus étoffée et mieux ajustée aux pratiques réelles des PME, à partir d'études empiriques ciblées et menées dans des secteurs d'activité comparables et homogènes, sur des échantillons d'entreprises de même taille. En fait, la probabilité d'obtenir des résultats peu significatifs demeure très élevée dans ce genre d'analyse: la trop forte diversité des entreprises enquêtées peut grandement réduire la qualité de l'interprétation des données recueillies. Par ailleurs, une autre précaution méthodologique pourrait résider dans le recours aux entrevues directes, de préférence à l'envoi de questionnaires par la poste, cette dernière méthode de cueillette de données s'avérant peu adaptée aux caractéristiques des propriétaires-dirigeants de PME.

Une autre série de questions pertinentes concerne les pistes de solutions inexplorées en GRH. Étant donné les particularités de leur environnement, les PME auraient-elles avantage à développer ou à adapter leurs pratiques de conservation des RH? Dans une telle perspective, nous pourrions suggérer des adaptations innovatrices de pratiques existantes ou carrément l'élaboration de pratiques nouvelles mieux adaptées aux contraintes inhérentes au contexte des PME. Cette démarche ne consisterait plus seulement à expliquer l'existant en GRH, mais également à identifier le souhaitable. Par analogie avec l'évolution des connaissances relatives aux grandes entreprises, on pourrait envisager ici des solutions s'inscrivant dans de nouveaux paradigmes de gestion, de la même façon que le mouvement de gestion participative est venu ébranler et remettre en question le paradigme taylorien de l'organisation scientifique du travail. 


\section{Bibliographie}

AMBA-RAO, S.C. et D. PENDSE (1985), «Human resource compensation and maintenance practices », American Journal of Small Business, vol. 10, ${ }^{\circ} 2$, automne, p. 19-29.

AudET, M., L. BÉLANGER et F. JACQUES (1987), Place, importance et pratiques de la gestion des ressources humaines dans les entreprises québécoises, Document de recherche, Collection instruments de travail, Département des relations industrielles, Université Laval, Québec, 105 p.

BACon, J. (1970), Executive Compensation Plans in the Smaller Company, Report $\mathrm{n}^{\circ}$ 15, Managing the Moderate-sized Company Series, National Industrial Conference Board, New York, 27 p.

BAGBY, D.R. (1987), «Employee benefits in small firms », dans R.G. Wyckham, L.N. Meredith et G.R. Bushe (éds), The Spirit of Entrepreneurship, Actes de la 32 Annual World Conference of the ICSB (Vancouver), Simon Fraser University, Burnaby, 10-12 juin, p. 278-286.

Baker, A.W. (1955), Personnel Management in Small Plants: A Study of Small Manufacturing Establishments in Ohio, Bureau of Business Research, College of Commerce and Administration, The Ohio State University, Colombus, 288 p.

BARRon, J.M., D.A. BlACK et M.A. Loewenstein (1987), «Employer size: the implications for search, training, capital investment, starting wages, and wage growth », Journal of Labor Economics, vol. 5, n 1, p. 76-89.

BAYAD, M. et J.L. HERRMANN (1992), «Panel des PMI Lorraines : rapport technique de la troisième vague. Enquête 1991 », Centre régional de documentation en gestion, Nancy, janvier.

Beaucourt, C., F. Zaddem et C. Mahieu (1989), «Industrial relations in small and medium-sized enterprises in France», dans P. Auer et H. Fehr-Duda (éds), Industrial Relations in Small and Medium-sized Enterprises - Final Report, Enterprise Policy Document, Office for Official Publications of the European Communities, Brussels - Luxembourg, p. 99-141.

Beaumont, P.B. et B. Townley (1985), «Greenfield sites, new plants and work practices », dans V. Hammond (éd.), Current Research in Management, Londres, Frances Pinter, p. 173.

Bélanger, L., C. Benabou, J.L. Bergeron, R. Foucher et A. Petit (1988), Gestion stratégique des ressources humaines, Montréal, Gaëtan Morin Éditeur, 662 p.

BENOIT, C. et M.D. RousseAu (1990), «La gestion des ressources humaines dans les petites et moyennes entreprises au Québec », Revue Internationale P.M.E., vol. 3, $\mathrm{n}^{\circ} 1$, automne, p. 39-55. 
BENOIT, C. et M.D. RousseAu (1993), La gestion des ressources humaines dans les $P M E$ au Québec: perception des dirigeants, [pour le] ministère de la Maind'œuvre, de la Sécurité du revenu et de la Formation professionnelle, Direction de la recherche, Publications du Québec, 293 p.

BRown, C. et J.L. MEdoff (1989), «The employer size-wage effect», Journal of Political Economy, vol. 97, n 5, p. 1027-1059.

CAMPBELL, A. (1989), «Industrial relations in small and medium-sized enterprises in the United Kingdom », dans P. Auer et H. Fehr-Duda, op. cit., p. 251-285.

CATHERINE, J. (1990), «La fonction personnel dans les établissements ou entreprises de 200 à 2000 personnes », Les Nouveaux Cahiers ANDCP, Paris, $\mathrm{n}^{\circ} 40$, mars, p. 17-34.

CÉGOS (1987), «Enquête CÉGOS : les syndicats dans les P.M.E. en 1985 », Personnel (Revue ANDCP), Paris, $\mathrm{n}^{\circ}$ 283, janvier, p. 21-26.

CHRISTIANSEN, R.O. (1980), «Impact of employee stock ownership plans on employee morale », American Journal of Small Business, vol. 5, $\mathrm{n}^{\circ}$ 1, juillet-sept, p. 22-31.

CoOley, P.L. et C.E. EDWARdS (1985), «Age effects on managerial compensation in small firms », American Journal of Small Business, vol. 10, $n^{\circ} 1$, été, p. 4.1-48.

D'AMBoISE, G. et D.J. GARAND (1993), Identification des difficultés et besoins des PME en matière de $G R H$, Rapport de recherche inédit, pour la Société québécoise de développement de la main-d'œuvre (SQDM), Montréal, octobre, 125 p.

D'Amboise, G. et Y. Gasse (1980), Performance in Small Firms and the Utilization of Formal Management Techniques, Document spécial, Faculté des sciences de l'administration, Université Laval, Québec, mai, n 80-17.

DANIEL, W.W. et N. MILlWARD (1985), Workplace Industrial Relations in Britain: The DE / PSI / ESRC Survey, Aldershot, UK, Gower Publishing Co., 338 p.

Dolan, S.L., V.P. Hogue et J. Harbottle (1990), "L'évolution des tendances en gestion des ressources humaines au Québec: étude comparative en fonction des tailles des entreprises », dans R. Blouin (éd.), Vingt-cinq ans de pratique en relations industrielles au Québec, Cowansville, Éditions Yvon Blais, p. 775-789.

Donnadieu, G. (1987), « Du nouveau dans les rémunérations », Personnel (Revue $A N D C P$ ), Paris, $\mathrm{n}^{\circ} 285$, mars-avril, p. 16-20.

EVANS, D.S. et L.S. LEIGHTON (1989), «Why do smaller firms pay less?», The Journal of Human Resources, vol. XXIV, nº 2, p. 299-318.

FABI, B. et D.J. GARAND (1993a), «L'acquisition des ressources humaines en PME», Revue Internationale P.M.E., vol. 6, $\mathrm{n}^{\mathrm{os}} 3 / 4$ (à paraître).

FABI, B. et D.J. GARAND (1994), «La gestion des ressources humaines en PME», dans P.A. Julien et al. (éd.), Les PME: bilan et perspectives, Québec, Presses Inter Universitaires, et Paris, Économica, p. 298-368. 
FABI, B., D.J. GARAND et N. PETTERSEN (1993), «La gestion des ressources humaines en PME: proposition d'un modèle de contingence », dans M. Amami et al. (éds), Compétitivité des PME et marchés sans frontière, Congrès international francophone de la PME, Carthage, Tunisie, 28-30 octobre, p. 216-229.

FAY, C.H. et S.D. MAURER (1986), «Developing compensation programs for the small business », dans H. Buckman Stephenson (éd.), The Impact of the Eighties on Small Business: Trends and Issues, Actes de la $10^{\mathrm{e}}$ Conférence annuelle de la SBIDA, Washington, D.C., p. 267-274.

GARAND, D.J. (1992), «Synthèse des pratiques d'acquisition des ressources humaines en PME », dans P. Louart et al. (éd.), La GRH avec ou sans frontières, Actes du $3^{\mathrm{e}}$ Congrès de l'AGRH, Lille, p. 412-425.

GARAND, D.J. (1993), Les pratiques de gestion des ressources humaines (GRH) en petites et moyennes entreprises (PME): une synthèse conceptuelle et empirique, Rapport de recherche, Groupe de recherche en économie et gestion des PME (GREPME), mars, 386 p.

GARAND, D.J. et B. FABI (1991), «Fondements conceptuels des pratiques de GRH en petites et moyennes entreprises (PME): formalisation, vision entrepreneuriale et modèle contingentiel ", dans A. Bernard et al. (éd.), Pour une vision de la GRH, Actes du $2^{\mathrm{e}}$ Congrès de l'AGRH, Cergy Pontoise, p. 324-336.

GARAND, D.J. et B. FABI (1992), «État de la recherche. Les pratiques de gestion des ressources humaines (GRH) en PME», Revue Organisation, vol. 2, n 1, automne, p. 61-99.

GARAND, D.J. et B. FABI (1993), «Les pratiques de conservation des ressources humaines en PME», dans G. Trepo et al. (éd.), Universalité et contingence de la $G R H$, Actes du $4^{\mathrm{e}}$ Congrès de l'AGRH, HEC-Paris, Jouy-en-Josas, p. 506-523.

GunNigle, P. et T. BRADY (1984), «The management of industrial relations in the small firm », Employee Relations, vol. 6, $\mathrm{n}^{\circ}$ 5, p. 21-24.

GUSKI, H.G. et H.J. SCHNEIDER (1977), Betriebliche Vermögensbildung in der Bundesrepublik Deutschland, Cologne, Allemagne.

HARRELL, T.W. (1970), «The personality of high earning MBA's in small business », Personnel Psychology, vol. 23, $\mathrm{n}^{\circ}$ 3, automne, p. 369-375.

HARRELl, T.W. (1971), «Differences between men in big and small business », Personnel Psychology, vol. 24, $\mathrm{n}^{\circ} 4$, hiver, p. 649-652.

HofFMAN, K. (1986), «Opinions of small Kansas business owners / operators concerning hiring, training, and advancement of male / female non-traditional workers in Kansas », dans H. Buckman Stephenson (éd.), op. cit., p. 261-266.

HOLLEY, W.H. JR. et R.S. WOLTERS (1987), «Employment at-will : an emerging issue for small businesses », Journal of Small Business Management, vol. 25, $\mathrm{n}^{\circ} 4$, octobre, p. 1-8. 
HORNSBY, J.S. et D.F. KURATKO (1990), "Human resource management in small business : critical issues for the 1990's », Journal of Small Business Management, vol. $28, \mathrm{n}^{\circ} 3$, juillet, p. 9-18.

HoRnsby, J.S., D.F. KuRATKo et D.W. NAFFZIGER (1992), «Flexible benefits : supporting the use of human resources as a distinctive competency for small business », Communication inédite, $37^{\mathrm{e}}$ Congrès international du CIPE/ICSB, Toronto, 18-21 juin, $13 \mathrm{p}$.

IDSON, T.L. et D.J. FEASTER (1990), «A selectivity model of employer-size wage differentials », Journal of Labor Economics, vol. 8, $\mathrm{n}^{\circ}$ 1, p. 99-122.

Ingham, G.K. (1970), Size of Industrial Organisation and Worker Behaviour, Cambridge, Cambridge University Press.

KRUSE, D. (1992), «Supervision, working conditions, and the employer size-wage effect », Industrial Relations, vol. 31, n² 2, p. 229-249.

LAROCHE, G. (1989), Petites et moyennes entreprises au Québec : organisation économique, croissance de l'emploi et qualité de vie au travail, Série de recherche $\mathrm{n}^{\circ} 91$, Institut international d'études sociales, Genève, 84 p.

LATONA, J.C. (1979), «Participative management in the small firm: a longitudinal study of an M.B.O. program », American Journal of Small Business, vol. III, $n^{\circ} 4$, avril, p. $15-22$.

LATONA, J.C. (1981), «Flexitime and the compressed workweek for the small firm: some employee reactions », American Journal of Small Business, vol. V, $\mathrm{n}^{\circ} 3$, janv.-mars, p. 23-29.

Le Louarn, J.Y. et R. Thériault (1984), Politiques et pratiques en recrutement et sélection en personnel dans les organisations au Québec, Association des professionnels en ressources humaines du Québec (APRHQ), juillet, Montréal, 79 p.

LESTER, R.A. (1967), «Pay differentials by size of establishment », Industrial Relations, vol. $7, \mathrm{n}^{\circ} 1$, octobre, p. 57-67.

LITTLE, B.L. (1986), « The performance of personnel duties in small Lousiana firms : a research note», Journal of Small Business Management, vol. 24, ${ }^{\circ} 4$, octobre, p. 66-69.

MAHÉ DE Boislandelle, H. (1988), Gestion des ressources humaines dans les P.M.E., Paris, Économica, Collection «Techniques de Gestion», 322 p.

MAHÉ DE Boislandelle, H. (1990), «Repérage de la fonction ressources humaines en P.M.E. », Revue Internationale P.M.E., vol. 3, $\mathrm{n}^{\circ}$ 1, automne, p. 27-37.

MAHÉ DE Boislandelle, H. (1993), Gérer les hommes de la jeune entreprise, Paris, Chotard Éditeurs, 200 p.

MAHÉ DE BoISLANDElle, H. et al. (1985), «La pratique de la gestion du personnel dans les P.M.E.», Rapport de recherche, CEGERH / FNEGE / IUT, Montpellier, janvier, $150 \mathrm{p}$. 
MAlaise, N. et V. De KeYSER (1988), Conditions de travail et petites et moyennes entreprises: Belgique, Fondation européenne pour l'amélioration des conditions de vie et de travail, Loughlinstown House, Shankill, Co. Dublin, Irlande, 80 p.

MARLOW, S. et D. PATTON (1993), "Managing the employment relationship in the smaller firm: possibilities for human resource management », Note de recherche, International Small Business Journal, vol. 11, n 4, p. 57-64.

MCEvoY, G.M. (1983), "Personnel practices in smaller firms: a survey and recommendations », American Journal of Small Business, vol. VIII, $\mathrm{n}^{\circ} 2$, oct.-déc., p. 32-39.

MCEvoy, G.M.(1984), «Small business personnel practices », Journal of Small Business Management, vol. 22, $\mathrm{n}^{\circ} 4$, p. 1-8.

MealieA, L.W. et D. LeE (1980), «Contemporary personnel practices in Canadian firms : an empirical evaluation », Relations Industrielles, vol. $35, \mathrm{n}^{\circ} 3$, p. 410-421.

Miguelez, F. (1989), «Industrial relations in small and medium-sized enterprises in Spain », dans P. Auer et H. Fehr-Duda, op. cit., p. 211-250.

MORISSETTE, R. (1993), «Canadian jobs and firm size : Do smaller firms pay less?», Canadian Journal of Economics / Revue Canadienne d'Économique, vol. XXVI, $\mathrm{n}^{\circ} 1$, p. $159-174$.

NEBENHAUs, D. (1987), «La participation aux décisions dans une PME. Étude de cas », Série Sciences de gestion, Économie et Sociétés, n 12, décembre, p. 111-137.

Neiswander, D.K., B.J. Bird et P.L. Young (1990), «Embauche et gestion des premiers employés d'une petite entreprise », Revue Internationale P.M.E., vol. 3, $\mathrm{n}^{\circ} 1$, automne,p. 7-25.

OCDE, (1985), «Employment in small and large firms: Where have the jobs come from?», Employment Outlook, septembre, p. 64-82.

PAumier, A. et D. GouAdain (1984), «La gestion prévisionnelle des ressources humaines : une enquête auprès d'un échantillon d'entreprises régionales », Direction et Gestion, $\mathrm{n}^{\circ}$ 6, p. 53-68.

Peters, V. (1974), Empirische Untersuchung über Arbeitszufriedenheit in einem Handweirksbetrieb, étude inédite, Munich, Allemagne.

PLEITNER, H.J. (1982), «Facteurs propres à influencer la satisfaction au travail dans la petite entreprise: un exemple empirique», dans Approvisionnements et Services Canada (éd.), Recueil des exposés: Le huitième congrès international de la petite entreprise, Ottawa, 20 octobre 1981, p. 213-225.

RAM, M. (1991), "The dynamics of workplace relations in small firms », International Small Business Journal, vol. 10, $\mathrm{n}^{\circ}$ 1, p. 44-53.

RINDT, K.E. (1975), «Small-firm personnel problems and management assistance», Journal of Small Business Management, vol. 13, $\mathrm{n}^{\circ}$ 3, juillet, p. 13-17. 
ROGER, A. (1985), Le suivi et l'appréciation du personnel dans les P.M.E. de services, Notes de recherche de l'I.A.E. d'Aix-en-Provence, CNRS 1984 Programme de recherche «Activités de services et $\mathrm{PME} », \mathrm{n}^{\circ} 308$, mai, $84 \mathrm{p}$ et $71 \mathrm{p}$.

SCOTT, M.G., I. Roberts, G. Holroyd et D. SAwbridge (1990), Management and Industrial Relations in Small Firms, Research Paper, UK Department of Employment, London, $\mathrm{n}^{\circ} 70$.

SHAW, K.N. et J.R. LANG (1985), « Executive compensation in emerging hi-tech industries », dans J.E. Brothers (éd.), The Challenge of Small Business Management Assistance Unification, Actes de la $9^{\mathrm{e}}$ Conférence annuelle de la SBIDA, San Diego, CA, 7-10 février, p. 389-393.

SUTTON, N.A. (1986), «A comparison of insurance and pension plans in large and small firms », American Journal of Small Business, vol. 11, $\mathrm{n}^{\circ} 2$, automne, p. 15-22.

THACKER, J.W. et R.J. CATTANEO (1987), «The Canadian personnel function: status and practices », dans T.H. Stone et J.Y. Le Louarn (éds.), Rapport du Congrès annuel du Groupe d'intérêt particulier sur le personnel et les ressources humaines de l'Association des sciences administratives du Canada (ASAC), Toronto, 1-3 juin, p. 56-66.

THÉRIAUlt, R. (1986), Politiques et pratiques en matière de rémunération globale dans les entreprises au Québec, Montréal, Les Productions Infort inc, novembre, $185 \mathrm{p}$.

ThÉriault, R. et J.Y. Le LouARn (1984), Politiques et pratiques en évaluation de la performance du personnel dans les organisations au Québec, Association des professionnels en RH du Québec (APRHQ), Montréal, 87 p.

Venet, P. (1988), «La fonction personnel dans les P.M.E. des Alpes-Maritimes et de Monaco», Personnel (Revue ANDCP), Paris, $\mathrm{n}^{\circ} 299$, octobre, p. 17-21.

VERSER, T.G. (1987), «Responses of inexperienced small business owners to perceived personnel problems », dans R.G. Wyckham, L.N. Meredith et G.R. Bushe, op. cit., p. 287-296.

WASSERMANN, W. (1989), «Industrial relations in small and medium-sized enterprises in the Federal Republic of Germany », dans P. Auer et H. Fehr-Duda, op. cit., p. 143-174.

WEISS, A. et H.J. LANDAU (1984), «Wages, hiring standards, and firm size », Journal of Labor Economics, vol. 2, n 4, p. 477-499. 